\title{
ORDERS OF NIKSHYCH'S HOPF ALGEBRA
}

\author{
JUAN CUADRA AND EHUD MEIR
}

\begin{abstract}
Let $p$ be an odd prime number and $K$ a number field having a primitive $p$-th root of unity $\zeta_{p}$. We prove that Nikshych's non group-theoretical Hopf algebra $H_{p}$, which is defined over $\mathbb{Q}\left(\zeta_{p}\right)$, admits a Hopf order over the ring of integers $\mathcal{O}_{K}$ if and only if there is an ideal $I$ of $\mathcal{O}_{K}$ such that $I^{2(p-1)}=(p)$. This condition does not hold in a cyclotomic field. Hence this gives an example of a semisimple Hopf algebra over a number field not admitting a Hopf order over any cyclotomic ring of integers. Moreover, we show that, when a Hopf order over $\mathcal{O}_{K}$ exists, it is unique and we describe it explicitly.
\end{abstract}

\section{INTRODUCTION}

Many results in the Representation Theory of Finite Groups exploit the fact that the complex group algebra $\mathbb{C} G$ of a finite group $G$ is defined over the integers or, more generally, over the ring of integers $\mathcal{O}_{K}$ of a number field $K$. In other terms, $\mathcal{O}_{K} G$ is an algebra order of $\mathbb{C} G$; indeed a Hopf (algebra) order. A prominent role is played by cyclotomic fields: for example, the celebrated Brauer's splitting field theorem states that any irreducible representation of $K G$ can be realized in $K(\omega)$, with $\omega$ a primitive root of unity of order equals $\exp G$ (see [3, Theorem 15.16, Corollary 15.18]).

Kaplansky's sixth conjecture, still unsolved, is a generalization of Frobenius Theorem for groups. It asserts that in a complex semisimple Hopf algebra $H$ the dimension of every irreducible representation of $H$ divides the dimension of $H$. Larson gave a positive answer in [6] if $H$ admits a Hopf order over a number ring. Motivated by this result, in [1] we addressed the question as to whether any complex semisimple Hopf algebra admits a Hopf order over a number ring. In the dimensions less than 36 in which the classification is complete ( 24 and 32 are still open) it turns out that all semisimple Hopf algebras are defined over cyclotomic rings of integers, see [1, Subsection 2.4] for an account. However, we exhibited in [1] an example in dimension 36 that does not admit a Hopf order over any number ring, although it satisfies the conjecture.

As a continuation of our previous work we investigate in this paper the problem of definability of semisimple Hopf algebras over cyclotomic ring of integers. Let $H$ be a semisimple Hopf algebra over a number field $K$ and suppose that $H$ has a Hopf order over some number ring. Does $H$ admit a Hopf order over a cyclotomic ring of integers contained in $K$ ? Our main result gives a negative answer for the family of non group-theoretical semisimple Hopf algebras $\left\{H_{p}\right\}$, with $p$ an odd prime, constructed by Nikshych (see [11]). The dimension of $H_{p}$ is $4 p^{2}$ (so in particular the dimension of $\mathrm{H}_{3}$ is 36). These Hopf algebras were not constructed explicitly but 
through a tensor category and a fiber functor. The representation category $\operatorname{Rep}\left(H_{p}\right)$ was obtained by equivariantization by $C_{2}$ from $\operatorname{Rep}\left(A_{p}\right)$, with $A_{p}$ the Hopf algebra studied by Masuoka in [7]. Using Tannaka reconstruction, in Section 3 we describe $H_{p}$ completely as follows:

Theorem 1. Let $\zeta_{p} \in \mathbb{C}$ be a primitive $p$-th root of unity. The Hopf algebra $H_{p}$ is generated, as an algebra over $\mathbb{C}$, by the elements $e_{0}, e_{1}, u_{a}, u_{b}, v_{a}, v_{b}$ and $g$ subject to the following relations:

$$
\begin{array}{llll}
e_{0}+e_{1}=1, & e_{0} e_{1}=e_{1} e_{0}=0, & & \\
u_{a}^{p}=u_{b}^{p}=e_{0}, & e_{0} u_{a}=u_{a}, & e_{0} u_{b}=u_{b}, & u_{a} u_{b}=u_{b} u_{a}, \\
v_{a}^{p}=v_{b}^{p}=e_{1}, & e_{1} v_{a}=v_{a}, & e_{1} v_{b}=v_{b}, & v_{a} v_{b}=\zeta_{p} v_{b} v_{a}, \\
g^{2}=1, & g u_{a}=u_{b} g, & g u_{b}=u_{a} g, & g v_{a}=v_{a} g, \quad g v_{b}=v_{b} g .
\end{array}
$$

The comultiplication, counit, and antipode of $H_{p}$ is given by the following formulas:

$$
\begin{array}{lll}
\Delta\left(u_{a}\right)=u_{a} \otimes u_{a}+v_{a} \otimes v_{a}, & \varepsilon\left(u_{a}\right)=1, & S\left(u_{a}\right)=u_{a}^{p-1}, \\
\Delta\left(u_{b}\right)=u_{b} \otimes u_{b}+v_{b} \otimes v_{b}^{p-1}, & \varepsilon\left(u_{b}\right)=1, & S\left(u_{b}\right)=u_{b}^{p-1}, \\
\Delta\left(v_{a}\right)=u_{a} \otimes v_{a}+v_{a} \otimes u_{a}, & \varepsilon\left(v_{a}\right)=0, & S\left(v_{a}\right)=v_{a}^{p-1} \\
\Delta\left(v_{b}\right)=u_{b} \otimes v_{b}+v_{b} \otimes u_{b}^{p-1}, & \varepsilon\left(v_{b}\right)=0, & S\left(v_{b}\right)=v_{b} .
\end{array}
$$

The comultiplication of $g$ is given by

$$
\begin{aligned}
\Delta(g)= & \frac{1}{p^{2}} \sum_{i, j, k, l} \zeta_{p}^{k j-i l} g u_{a}^{i} u_{b}^{j} \otimes g u_{a}^{k} u_{b}^{l}+\frac{1}{p} \sum_{k, l} \zeta_{p}^{-(k+l) k} g u_{a}^{k} u_{b}^{l} \otimes g v_{a}^{k+l} v_{b}^{k+l} \\
& +\frac{1}{p} \sum_{k, l} \zeta_{p}^{k(k+l)} g v_{a}^{k+l} v_{b}^{(p-1)(k+l)} \otimes g u_{a}^{k} u_{b}^{l}+\frac{1}{p} \sum_{k, l} g v_{a}^{k} v_{b}^{l} \otimes g v_{a}^{(p-1) l} v_{b}^{k} .
\end{aligned}
$$

The counit and antipode of $g$ are $\varepsilon(g)=1$ and $S(g)=g$.

In Section 4 we delve into the structure of $H_{p}$ : we describe its irreducible (co)representations and attached (co)characters, its Hopf automorphisms, and we show that $H_{p}$ is self-dual.

The set

$$
\mathcal{B}:=\left\{u_{a}^{i} u_{b}^{j}\right\} \cup\left\{v_{a}^{i} v_{b}^{j}\right\} \cup\left\{g u_{a}^{i} u_{b}^{j}\right\} \cup\left\{g v_{a}^{i} v_{b}^{j}\right\}
$$

is a basis of $H_{p}$. All structure constants of $H_{p}$ in this basis belong to $\mathbb{Q}\left(\zeta_{p}\right)$. Hence $H_{p}$ is defined over $\mathbb{Q}\left(\zeta_{p}\right)$. Our main result states:

Theorem 2. Let $K$ be a number field containing a primitive p-th root of unity $\zeta_{p}$. Consider $H_{p}$ as defined over $K$. Then, $H_{p}$ admits a Hopf order over $\mathcal{O}_{K}$, which must be unique, if and only if there is an ideal $I$ of $\mathcal{O}_{K}$ such that $I^{2(p-1)}=(p)$. In particular, $K$ can not be a cyclotomic field (nor an abelian extension of $\mathbb{Q}$ ) if a Hopf order exists.

This theorem implies that Nikshych's Hopf algebras behave rather differently than group algebras. Firstly, all group algebras are already defined over $\mathbb{Z}$. Secondly, the number of Hopf orders of a group algebra over $\mathcal{O}_{K}$ depends on $K$, and in some cases it is not bounded (see for example the classification of orders of the group algebras of the cyclic groups of prime orders in Section 2). 
The main result is contained in Section 5. We outline the strategy to prove it and construct the Hopf order. The element $h:=u_{a}+v_{a}$ is a group-like element of $H_{p}$ and generate a Hopf subalgebra isomorphic to $K C_{p}$. If $X$ is a Hopf order of $H_{p}$ over $\mathcal{O}_{K}$, then $X \cap K C_{p}$ is a Hopf order of $K C_{p}$. The Hopf orders of the latter are known by the works of Greither, Larson, Tate and Oort (we review their description in Section 2, after the preliminaries). They are given by ideals $I$ of $\mathcal{O}_{K}$ containing $\zeta_{p}-1$, see Formula 2.1. Denoting by $H(I)$ the corresponding Hopf order, the $\mathcal{O}_{K}$-submodule of integrals of $H(I)$ is $\frac{1}{p} I^{p-1} \sum_{i} h^{i}$. This determines uniquely the Hopf orders of $K C_{p}$. On the other hand, any Hopf order must contain certain elements arising from characters and cocharacters. The proof of the main result is based on the interaction between the order $X$ of $H_{p}$ and the order $X \cap K C_{p}$ of $K C_{p}$. We exhibit certain elements which must be in $X$. We then conclude that necessarily $\frac{1}{\sqrt{p}} \sum_{i} h^{i} \in X \cap K C_{p}$, and by the classification of orders mentioned above, we conclude that some more elements must lie in $X \cap K C_{p}$ and therefore in $X$. We then show that these elements generate an order of $H_{p}$, which thus must be a minimal order. We then use the self-duality of $H_{p}$ and conclude that there is also a maximal order. A result of Larson (see Proposition 1.4) now implies that the two orders must be equal, and therefore we only have one order. The necessity of the existence of an ideal $I$ of $\mathcal{O}_{K}$ such that $I^{2(p-1)}=(p)$ arises from the following consideration: We prove that the set of integrals of $X \cap K C_{p}$ is exactly $\mathcal{O}_{K}\left(\frac{1}{\sqrt{p}} \sum_{i} h^{i}\right)$. We write $J=\{x \in K \mid x(h-1) \in X\}$. By the classification in Section 2 we find out that $I:=J^{-1}$ must satisfy $I^{2(p-1)}=(p)$. The unique Hopf order of $H_{p}$ is the $\mathcal{O}_{K}$-subalgebra of $H_{p}$ generated by $e_{0}, e_{1}, g, J\left(u_{a}-e_{0}\right), J\left(u_{b}-e_{0}\right), J\left(v_{a}-e_{1}\right)$, and $J\left(v_{b}-e_{1}\right)$.

In Section 6 we study the problem of definability over cyclotomic ring of integers of $H_{p}$ but now considered as a complex Hopf algebra. Since $H_{p}$ is already defined over a number field $K$, the question reads now as follows. Let $L / K$ be a Galois extension. Could a $L / K$-form of $H_{p}$ admit an order over some cyclotomic ring of integers? Namely, could there be another Hopf algebra $H_{p}^{\prime}$ over $K$ such that $H_{p}^{\prime} \otimes_{K} L \simeq H_{p} \otimes_{K} L$ and $H_{p}^{\prime}$ admits an order over some cyclotomic ring of integers? The following result gives a number theoretical condition under which the answer is affirmative:

Theorem 3. Let $\zeta_{n} \in \mathbb{C}$ be a primitive $n$-th root of unity, with $n$ divisible by $p$. Consider $H_{p}$ as defined over $\mathbb{Q}\left(\zeta_{n}\right)$. Let $w \in \mathbb{Z}\left[\zeta_{n}\right]$ and $t \in \mathbb{C}$ be such that $w$ is invertible and $t^{2}=w\left(\zeta_{p}-1\right)$. Assume that there is $d \in \mathbb{Z}\left[\zeta_{n}\right]$ such that $\frac{1}{2}(d+t) \in$ $\mathcal{O}_{\mathbb{Q}\left(\zeta_{n}, t\right)}$. Then, $H_{p}$ admits a $\mathbb{Q}\left(\zeta_{n}, t\right) / \mathbb{Q}\left(\zeta_{n}\right)$-form $H_{p}^{\prime}$ which in turn admits an order over $\mathbb{Z}\left[\zeta_{n}\right]$.

For $p=7$ and $n=28$ we construct elements $w, t$ and $d$ satisfying this condition. So, $H_{7}$, as a complex Hopf algebra, admits an order over the cyclotomic ring of integers $\mathbb{Z}\left[\zeta_{28}\right]$.

The following questions on the definability over cyclotomic ring of integers of complex semisimple Hopf algebras remain open:

Questions. Does there exist a value of $p$ for which Nikshych's Hopf algebra $H_{p}$, as defined over the complex numbers, does not admit an order over any cyclotomic ring 
of integers? More generally, does there exist a complex semisimple Hopf algebra which admits an order over a number ring but not over any cyclotomic ring of integers?

\section{PReliminaries}

Throughout $H$ is a finite-dimensional Hopf algebra over a ground field $K$. Unless otherwise stated, vector spaces, linear maps, and unadorned tensor products are over $K$. The identity element of $H$ is denoted by $1_{H}$ and the comultiplication, counit, and antipode by $\Delta, \varepsilon$, and $S$ respectively. Our main references for Hopf algebra theory are [9] and [12].

We next collect from [1, Subsection 1.2] several notions and results on Hopf orders that we will need later. We refer the reader to there for the proofs.

1.1. Hopf orders. Let $R \subset K$ be a subring and $V$ a finite-dimensional $K$-vector space. Recall that an order of $V$ over $R$ is a finitely generated and projective $R$ submodule $X$ of $V$ such that the natural map $X \otimes_{R} K \rightarrow V$ is an isomorphism. We view $X$ inside $V$ as the image of $X \otimes_{R} R$. A Hopf order of $H$ over $R$ is an order $X$ of $H$ such that $1_{H} \in X, X X \subseteq X, \Delta(X) \subseteq X \otimes_{R} X, \varepsilon(X) \subseteq R$ and $S(X) \subseteq X$. (Note that $X \otimes_{R} X$ can be identified naturally as an $R$-submodule of $H \otimes H$.) Equivalently, a Hopf order of $H$ over $R$ is a Hopf algebra $X$ over $R$, which is finitely generated and projective as an $R$-module, such that $X \otimes_{R} K \simeq H$ as Hopf algebras over $K$. We will assume throughout this subsection that $K$ is a number field and $R=\mathcal{O}_{K}$. A Hopf order without indication of the ground ring means a Hopf order over $R$.

Proposition 1.1. Let $X$ be a Hopf order of $H$.

(i) The dual order $X^{\star}:=\left\{\varphi \in H^{*}: \varphi(X) \subseteq R\right\}$ is a Hopf order of $H^{*}$.

(ii) The natural isomorphism $H \simeq H^{* *}$ induces an isomorphism of Hopf orders $X \simeq X^{\star \star}$.

(iii) If $A$ is a Hopf subalgebra of $H$, then $X \cap A$ is a Hopf order of $A$.

(iv) If $f: H \rightarrow B$ is a surjective Hopf algebra map, then $f(X)$ is a Hopf order of $B$.

An important fact in our study of Hopf orders is that they contain certain elements arising from the characters and cocharacters of the Hopf algebra.

Proposition 1.2. Let $X$ be a Hopf order of $H$. Any character of $H$ belongs to $X^{\star}$. As a consequence, any character of $H^{*}$ belongs to $X$.

We will also need the following two results by Larson:

Proposition 1.3. [6, Proposition 2.2] Let $H$ be a semisimple Hopf algebra over $K$ and $X$ a Hopf order of $H$. Denote by $\Lambda_{X}$ and $\Lambda_{X^{\star}}$ the $R$-submodule of left integrals of $X$ and $X^{\star}$ respectively. Then $\varepsilon\left(\Lambda_{X}\right) \varepsilon\left(\Lambda_{X^{\star}}\right)=(\operatorname{dim} H)$ as ideals in $R$.

Proposition 1.4. [6, Corollary 3.2] With hypotheses as before, assume that $X$ and $Y$ are Hopf orders of $H$ such that $X \subseteq Y$. If $\varepsilon\left(\Lambda_{X}\right)=\varepsilon\left(\Lambda_{Y}\right)$, then $X=Y$. 


\section{Classification of Hopf orders of $K C_{p}$}

Let $p$ be a prime number and $\zeta$ a primitive $p$-th root of unity. Let $K$ be a number field containing $\zeta$ and $R:=\mathcal{O}_{K}$. Let $\sigma$ denote a generator of the cyclic group $C_{p}$. We will describe here all Hopf orders of $K C_{p}$. Tate and Oort classified all group schemes of order $p$ over $R$ in [14, Theorem 3]. Their result is more general than classifying Hopf orders over $R$. However, we will combine it with Greither's result 4, Lemma 1.2, page 40] to give a more explicit description of all Hopf orders of $K C_{p}$.

We begin with the following observation:

Lemma 2.1. Let $X$ be a Hopf order of $K C_{p}$. Consider the fractional ideal

$$
J=\{\alpha \in K: \alpha(\sigma-1) \in X\} .
$$

Then $R \subseteq J \subseteq R \frac{1}{\zeta-1}$.

Proof. By Proposition 1.2, $\psi(X) \subseteq R$ for any character $\psi$ of $C_{p}$. Using the character mapping $\sigma$ to $\zeta$ we see that $J(\zeta-1) \subseteq R$. Hence $J \subseteq R \frac{1}{\zeta-1}$. For the other inclusion, notice that $\sigma$ is a character of $\left(K C_{p}\right)^{*}$. Then $\sigma \in X$ again by Proposition 1.2, and $R(\sigma-1) \subseteq X$.

The above lemma leads us to the following definition:

Definition 2.2. Let $I$ be an ideal of $R$ containing $\zeta-1$. The global Larson order associated to $I$ is the $R$-submodule of $K C_{p}$

$$
H(I)=\bigoplus_{i=0}^{p-1} I^{i}(\zeta-1)^{-i}(\sigma-1)^{i}
$$

The name global Larson order will make sense in a few paragraphs. Notice that if $(\zeta-1) \subseteq I \subseteq I^{\prime}$, then $H(I) \subseteq H\left(I^{\prime}\right)$. Even though the Larson orders are orders of the cyclic group algebra, they will play a decisive role in the classification of orders of Nikshych's Hopf algebra in Section 5 .

Lemma 2.3. The global Larson orders are Hopf orders of $K C_{p}$. The set of integrals in $H(I)$ is $\frac{1}{p} I^{p-1} \sum_{i} \sigma^{i}$.

Proof. We first show that $H(I)$ is closed under multiplication. For this, it is enough to prove that $I^{p}(\zeta-1)^{-p}(\sigma-1)^{p} \subseteq H(I)$. This follows from the fact that the element $x:=\frac{1}{\zeta-1}(\sigma-1)$ satisfies a monic polynomial over $R$ of degree $p$. We have:

$$
1=\sigma^{p}=((\zeta-1) x+1)^{p}=\sum_{k=0}^{p}\left(\begin{array}{l}
p \\
k
\end{array}\right)(\zeta-1)^{k} x^{k} \Longrightarrow \sum_{k=1}^{p}\left(\begin{array}{l}
p \\
k
\end{array}\right)(\zeta-1)^{k-1} x^{k}=0 .
$$

The coefficient of $x^{p}$ is $(\zeta-1)^{p-1}$ and this equals $p \xi$ for some $\xi \in R$ invertible. Multiplying by $p^{-1} \xi^{-1}$ we obtain the desired polynomial. On the other hand, it is clear that $1 \in H(I), \varepsilon(H(I)) \subseteq R$, and $S(H(I)) \subseteq H(I)$. It remains to prove that $\Delta(H(I)) \subset H(I) \otimes_{R} H(I)$. Since $\Delta$ is an algebra map and $H(I)$ is closed under multiplication, it suffices to check that $\Delta(r x) \in H(I) \otimes_{R} H(I)$ for every $r \in I$. A direct calculation reveals that

$$
\Delta(r x)=r x \otimes 1+1 \otimes r x+(\zeta-1) x \otimes r x .
$$


The first two summands clearly belong to $H(I) \otimes_{R} H(I)$ and the third summand too because $\zeta-1 \in I$.

To prove the statement about the integrals, notice that the integral $\frac{1}{p} \sum_{i} \sigma^{i}$ equals an invertible element times a monic polynomial $f$ of degree $p-1$ in $x$. This can be seen by the following calculation:

$$
\frac{1}{p} \sum_{i} \sigma^{i}=\frac{1}{p} \frac{((\zeta-1) x+1)^{p}-1}{(\zeta-1) x}=\sum_{k=1}^{p} \frac{1}{p}\left(\begin{array}{l}
p \\
k
\end{array}\right)(\zeta-1)^{k-1} x^{k-1} .
$$

The fractional expression is just symbolic as $(\zeta-1) x$ is not necessarily invertible. The powers of $x$ in the right-hand side term have coefficients in $R$. Observe that $p$ divides $\left(\begin{array}{l}p \\ k\end{array}\right)$ for $k=1, \ldots, p-1$. For $k=p$ the coefficient of $x^{p-1}$ is $(\zeta-1)^{p-1}=p \xi$ with $\xi \in R$ invertible. If $r \in I^{p-1}$, then $\frac{r}{p} \sum_{i} \sigma^{i}$ is an integral in $H(I)$ by (2.2), since $\zeta-1 \in I$. For the reverse inclusion, observe that by construction we have $I^{p-1}=\left\{\alpha \in K: \alpha x^{p-1} \in H(I)\right\}$. Let $\int$ be an integral in $H(I)$. There is $\lambda \in K$ such that $\int=\frac{\lambda}{p} \sum_{i} \sigma^{i}$. Then $\lambda \xi x^{p-1} \in H(I)$ by (2.2) and thus $\lambda \in I^{p-1}$.

We will next prove that all Hopf orders of $K C_{p}$ are global Larson orders. Over a local ring, this is a theorem by Greither, see [4, Lemma 1.2, page 40]. We will use the local to global result of Tate and Oort [14, Lemma 4] to pass to the number field case.

Let $\mathfrak{p} \subset R$ be a prime ideal such that $p \in \mathfrak{p}$. Consider the corresponding valuation $\nu$, scaled so that $\nu(p)=1$ (we find more convenient to write here the valuation in additive terms). Then it is easy to see that $\nu(1-\zeta)=\frac{1}{p-1}$ because $(\zeta-1)^{p-1}=(p)$.

Definition 2.4. [5, Section 3] Let $b \in R_{\mathfrak{p}}$ be such that $0 \leq \nu(b) \leq \frac{1}{p-1}$. Set $s=\nu(b)$. The Larson order $H(s)$ is the $R_{\mathfrak{p}}$-subalgebra of $K_{\mathfrak{p}} C_{p}$ generated by $\frac{1}{b}(\sigma-1)$.

One can see, exactly as in Lemma 2.3, that Larson orders are indeed Hopf orders, and that $H(s)$ does not depend on the choice of $b$. Notice that $H(s)$ is defined only if there is an element with valuation $s$ in $R_{\mathfrak{p}}$. We have the following classification result by Greither, see [15, Theorem 3.0.0] and [4, Lemma 1.2, page 40].

Theorem 2.5 (Greither). All Hopf orders of $K_{\mathfrak{p}} C_{p}$ over $R_{\mathfrak{p}}$ are Larson orders.

We recall the following result of Tate and Oort:

Proposition 2.6. 14, Lemma 4] For any commutative ring $T$, let $E(T)$ denote the set of isomorphism classes of group schemes of order $p$ over $T$. Then, the square

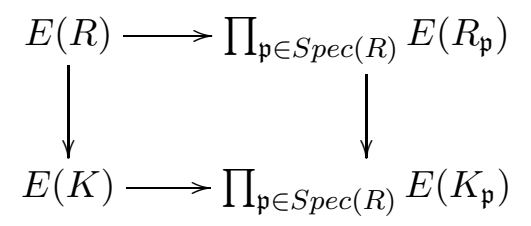

where the maps are given by extension of scalars, is cartesian.

With this in hand we can establish:

Theorem 2.7. Every Hopf order of $K C_{p}$ over $R$ is a global Larson order. 
Proof. A Hopf order $X$ of $K C_{p}$ over $R$ can be viewed as a group scheme of order $p$. Proposition 2.6 tells us that giving $X$ is the same as giving its extension of scalars to $K$ and $R_{\mathfrak{p}}$ for every $\mathfrak{p} \in \operatorname{Spec}(R)$, in a compatible way. The extension of scalars of $X$ to $K$ will be just $K C_{p}$, and thus we know the extension of scalars to all $K_{\mathfrak{p}}$. Furthermore, if $\mathfrak{p} \in \operatorname{Spec}(R)$ satisfies $p \notin \mathfrak{p}$, then we only have one Hopf order over $R_{\mathfrak{p}}$. This is because all primitive idempotents will be contained in any Hopf order.

The different orders will differ only by their extension of scalars to $R_{\mathfrak{p}}$ with $p \in \mathfrak{p}$. We know by Greither's Theorem that $X \otimes_{R} R_{\mathfrak{p}}$ is a Larson order over $R_{\mathfrak{p}}$. Let $\mathfrak{q}_{1}^{r_{1}} \cdots \mathfrak{q}_{l}^{r_{l}}$ be the prime decomposition of $(\zeta-1)$ in $R$. Assume that $X \otimes_{R} R_{\mathfrak{q}_{i}}$ is isomorphic to $H\left(s_{i}\right)$ over $R_{\mathfrak{q}_{i}}$. Consider the ideal $I=\prod_{i} \mathfrak{q}_{i}^{(p-1) r_{i} s_{i}}$. One can now see that the Larson order $H(I)$ will give rise to exactly the same localizations as $X$ at $\mathfrak{q}_{i}$. Since the square in Proposition 2.6 is cartesian, this means that $X=H(I)$.

We know how the integrals inside Larson orders look like by Lemma 2.3. As a consequence:

Corollary 2.8. A Hopf order $H(J)$ of $K C_{p}$ over $R$ which contains $\frac{1}{p} I^{p-1} \sum_{i} \sigma^{i}$ contains the Hopf order $H(I)$.

Proof. Using the prime decomposition of ideals, $I^{p-1} \subseteq J^{p-1}$ implies $I \subseteq J$.

The computation of the submodule of integrals in Lemma 2.3 together with Theorem 2.7 has the following outcome, from which we will derive the necessary condition in our main theorem:

Corollary 2.9. Let $X$ be a Hopf order of $K C_{p}$.

(i) Suppose that the $R$-submodule of integrals of $X$ is generated by $\frac{1}{\sqrt{p}} \sum_{i} \sigma^{i}$. Then there exists an ideal $I$ of $R$ such that $I^{2(p-1)}=(p)$.

(ii) Suppose that $\frac{1}{\sqrt{p}} \sum_{i} \sigma^{i} \in X$ and there is $\pi \in K$ such that $\pi^{2}=\zeta-1$. Then $\frac{1}{\pi}(\sigma-1) \in X$.

Proof. (i) In view of Theorem 2.7, $X$ is isomorphic to $H(I)$ for some ideal $I$ of $R$ containing $\zeta-1$. By hypothesis and Lemma 2.3 the submodule of integrals is

$$
R\left(\frac{1}{\sqrt{p}} \sum_{i} \sigma^{i}\right)=\frac{1}{p} I^{p-1} \sum_{i} \sigma^{i} .
$$

Then $I^{p-1}=(\sqrt{p})$ and thus $I^{2(p-1)}=(p)$.

(ii) From the hypothesis and Lemma 2.3 , we obtain $(\sqrt{p}) \subseteq I^{p-1}$. We know that $(\zeta-1)^{p-1}=(p)$. Using the prime factorization of ideals, we have $(\pi)^{p-1}=(\sqrt{p}) \subseteq$ $I^{p-1}$. This implies that $(\pi) \subseteq I$. Then the element $\frac{\pi}{\zeta-1}(\sigma-1)=\frac{1}{\pi}(\sigma-1) \in X$ by the construction of $H(I)$.

\section{An EXPlicit DESCRIPTION OF NiKshyCh's Hopf Algebra}

The goal of this section will be to write in an explicit way Nikshych's Hopf algebra.

For an odd prime number $p$, Nikshych constructed in [11] a finite-dimensional, semisimple, weakly group-theoretical and non group-theoretical Hopf algebra $H_{p}$ of dimension $4 p^{2}$. It was defined in terms of a tensor category and a fiber functor. The 
representation category $\operatorname{Rep}\left(H_{p}\right)$ is constructed from the representation category of another Hopf algebra, $A_{p}$, by means of equivariantization by $C_{2}$. As fusion categories, $\operatorname{Rep}\left(H_{p}\right) \simeq \operatorname{Rep}\left(A_{p}\right)^{C_{2}}$. The Hopf algebra $A_{p}$ first appeared in the work of Masuoka [7. The above equivalence implies that $H_{p}$ fits into the short exact sequence

$$
K \rightarrow A_{p} \rightarrow H_{p} \rightarrow K_{2} \rightarrow K .
$$

To describe explicitly the structure of $H_{p}$ we need to write the structure of $A_{p}$, the action of the generator $g$ of $C_{2}$ on $A_{p}$, and the comultiplication of $g$.

From now on we abbreviate $A_{p}$ to $A$ and $H_{p}$ to $H$. In this section we assume that $K$ is algebraically closed of characteristic zero.

The main result of this section is the following:

Theorem 3.1. Let $\zeta \in K$ be a primitive $p$-th root of unity. The Hopf algebra $H$ is generated, as an algebra over $K$, by the elements $e_{0}, e_{1}, u_{a}, u_{b}, v_{a}, v_{b}$ and $g$ subject to the following relations:

$$
\begin{array}{llll}
e_{0}+e_{1}=1, & e_{0} e_{1}=e_{1} e_{0}=0, & & \\
u_{a}^{p}=u_{b}^{p}=e_{0}, & e_{0} u_{a}=u_{a}, & e_{0} u_{b}=u_{b}, & u_{a} u_{b}=u_{b} u_{a}, \\
v_{a}^{p}=v_{b}^{p}=e_{1}, & e_{1} v_{a}=v_{a}, & e_{1} v_{b}=v_{b}, & v_{a} v_{b}=\zeta_{p} v_{b} v_{a}, \\
g^{2}=1, & g u_{a}=u_{b} g, & g u_{b}=u_{a} g, & g v_{a}=v_{a} g, \quad g v_{b}=v_{b} g .
\end{array}
$$

The comultiplication, counit, and antipode of $H$ is given by the following formulas:

$$
\begin{array}{lll}
\Delta\left(u_{a}\right)=u_{a} \otimes u_{a}+v_{a} \otimes v_{a}, & \varepsilon\left(u_{a}\right)=1, & S\left(u_{a}\right)=u_{a}^{p-1}, \\
\Delta\left(u_{b}\right)=u_{b} \otimes u_{b}+v_{b} \otimes v_{b}^{p-1}, & \varepsilon\left(u_{b}\right)=1, & S\left(u_{b}\right)=u_{b}^{p-1}, \\
\Delta\left(v_{a}\right)=u_{a} \otimes v_{a}+v_{a} \otimes u_{a}, & \varepsilon\left(v_{a}\right)=0, & S\left(v_{a}\right)=v_{a}^{p-1}, \\
\Delta\left(v_{b}\right)=u_{b} \otimes v_{b}+v_{b} \otimes u_{b}^{p-1}, & \varepsilon\left(v_{b}\right)=0, & S\left(v_{b}\right)=v_{b} .
\end{array}
$$

The comultiplication of $g$ is given by

$$
\begin{aligned}
\Delta(g)= & \frac{1}{p^{2}} \sum_{i, j, k, l} \zeta^{k j-i l} g u_{a}^{i} u_{b}^{j} \otimes g u_{a}^{k} u_{b}^{l}+\frac{1}{p} \sum_{k, l} \zeta^{-(k+l) k} g u_{a}^{k} u_{b}^{l} \otimes g v_{a}^{k+l} v_{b}^{k+l} \\
& +\frac{1}{p} \sum_{k, l} \zeta^{k(k+l)} g v_{a}^{k+l} v_{b}^{(p-1)(k+l)} \otimes g u_{a}^{k} u_{b}^{l}+\frac{1}{p} \sum_{k, l} g v_{a}^{k} v_{b}^{l} \otimes g v_{a}^{(p-1) l} v_{b}^{k} .
\end{aligned}
$$

The counit and antipode of $g$ are $\varepsilon(g)=1$ and $S(g)=g$.

The rest of this section will be devoted to prove Theorem 3.1 .

3.1. The algebra $A$. As an algebra, $A$ is the direct sum

$$
K\left(C_{p} \times C_{p}\right) \oplus K^{c}\left(C_{p} \times C_{p}\right),
$$

where $c:\left(C_{p} \times C_{p}\right) \times\left(C_{p} \times C_{p}\right) \rightarrow K^{\times}$is the 2-cocycle given by

$$
c\left(a^{i} b^{j}, a^{k} b^{l}\right)=\zeta^{-j k}, \quad 0 \leq i, j, k, l<p .
$$

Here $a, b$ are generators of $C_{p} \times C_{p}$. We present the group algebra $K\left(C_{p} \times C_{p}\right)$ by generators $u_{a}, u_{b}$ and defining relations $u_{a}^{p}=u_{b}^{p}=1, u_{a} u_{b}=u_{b} u_{a}$. The twisted group algebra $K^{c}\left(C_{p} \times C_{p}\right)$ is presented by generators $v_{a}, v_{b}$ and relations $v_{a}^{p}=v_{b}^{p}=$ $1, v_{a} v_{b}=\zeta v_{b} v_{a}$. Notice that $K^{c}\left(C_{p} \times C_{p}\right)$ is isomorphic to the matrix algebra $\mathrm{M}_{p}(K)$. 
To shorten, we set $A_{0}=K\left(C_{p} \times C_{p}\right)$ and $A_{1}=K^{c}\left(C_{p} \times C_{p}\right)$. We denote the units of $A_{0}$ and $A_{1}$ by $e_{0}$ and $e_{1}$ respectively. So $1_{A}=e_{0}+e_{1}$ and $e_{0} e_{1}=e_{1} e_{0}=0$. Unless otherwise specified, the inverses are taking inside either $A_{0}$ or $A_{1}$. For example, $u_{a}^{-1}$ means $u_{a}^{p-1}$.

The comultiplication, counit, and antipode of $A$ are described in (3.1) above.

3.2. The algebra $H$. As an algebra, $H$ is the crossed product $A * K C_{2}$, where $g$ acts as an algebra automorphism on $A$ by:

$$
g\left(u_{a}\right)=u_{b}, \quad g\left(u_{b}\right)=u_{a}, \quad g\left(v_{a}\right)=v_{a}, \quad g\left(v_{b}\right)=v_{b} .
$$

In $H$ we have the relations:

$$
g u_{a}=u_{b} g, \quad g u_{b}=u_{a} g, \quad g v_{a}=v_{a} g, \quad g v_{b}=v_{b} g .
$$

The hard part in the description of $H$ is the formula for $\Delta(g)$. Recall from [11 that $H$ is constructed as follows: the automorphism $g$ induces an autoequivalence

$$
F: \operatorname{Rep}(A) \rightarrow \operatorname{Rep}(A), V \mapsto{ }_{g} V .
$$

Here ${ }_{g} V=V$ as a vector space, with new action $x \cdot v=g(x) v$ for all $x \in A, v \in V$. The functor $F$ is a tensor equivalence. Moreover, $F^{-1}=F$. To compute $\Delta(g)$ we will need to describe the tensor structure of $F$. For this, we first need to consider the irreducible representations of $A$.

3.3. Irreducible representations of $A$. Every irreducible representation of $A$ is an irreducible representation of either $A_{0}$ or $A_{1}$.

The algebra $A_{0}$ has $p^{2}$ one-dimensional irreducible representations, which we denote by $K^{i, j}$ with $0 \leq i, j<p$. As a $K$-vector space, $K^{i, j}=K$. The action of $u_{a}$ and $u_{b}$ on $K^{i, j}$ is given by:

$$
u_{a} \cdot 1=\zeta^{i} 1 \quad u_{b} \cdot 1=\zeta^{j} 1 .
$$

The algebra $A_{1}$ has only one irreducible representation, of dimension $p$, which we denote by $M$. Let $\left\{m_{i}: 0 \leq i<p\right\}$ be a basis for $M$. The action of $A_{1}$ on $M$ is

$$
v_{a} \cdot m_{i}=\zeta^{i} m_{i}, \quad v_{b} \cdot m_{i}=m_{i+1} \text { (indices are taken mod. } p \text { ). }
$$

3.4. Tensor structure on $F$. For any $V, W \in \operatorname{Rep}(A)$ irreducible we must establish an isomorphism $\theta_{V, W}: F(V \otimes W) \rightarrow F(V) \otimes F(W)$ satisfying the unit and associativity constraints. We do need to calculate these isomorphisms explicitly, as we will use them later to compute $\Delta(g)$. Observe that at the level of representations $F\left(K^{i, j}\right)=K^{j, i}$ and $F(M)=M$.

3.4.1. Isomorphisms between certain representations of $A$. Given $x \in A_{1}$ invertible, ${ }_{x} M$ stands for the following representation of $A_{1}$ : as a vector space, ${ }_{x} M=M$, and the action is given by

$$
y \cdot m=\left(x^{-1} y x\right) m \quad \forall y \in A_{1}, m \in M .
$$

We have an isomorphism

$$
{ }_{x} M \rightarrow M, m \mapsto x m .
$$

This will be used in this subsection to define isomorphisms between certain tensor products of representation. Consider the representation $K^{i, j} \otimes M$. Identify it with $M$, as a vector space, via $1 \otimes m \mapsto m$. Under this identification, $v_{a}$ and $v_{b}$ act via 
$\zeta^{i} v_{a}$ and $\zeta^{j} v_{b}$ respectively. Since $v_{a} v_{b}=\zeta v_{b} v_{a}$, we see that this is the same as ${ }_{x} M$ for $x=v_{a}^{-j} v_{b}^{i}$. Then we have an isomorphism of representations

$$
l_{i, j}: K^{i, j} \otimes M \rightarrow M, 1 \otimes m \mapsto\left(v_{a}^{-j} v_{b}^{i}\right) m .
$$

In a similar fashion, $M \otimes K^{i, j}$ is isomorphic to ${ }_{x} M$ for $x=v_{a}^{j} v_{b}^{i}$ via

$$
r_{i, j}: M \otimes K^{i, j} \rightarrow M, m \otimes 1 \mapsto\left(v_{a}^{j} v_{b}^{i}\right) m .
$$

We discuss separately the four different cases that occur in the description of $\theta_{V, W}$ :

3.4.2. Two representations of $A_{0}$. We begin by considering the case $V=K^{i, j}$ and $W=K^{k, l}$. We have $V \otimes W \simeq K^{i+k, j+l}$. We must give an isomorphism between $F(V \otimes W) \simeq F\left(K^{i+k, j+l}\right) \simeq K^{j+l, i+k}$ and $F(V) \otimes F(W) \simeq K^{j, i} \otimes K^{l, k} \simeq K^{j+l, i+k}$. It will be determined by a nonzero scalar $\mu((i, j),(k, l))$. Then:

$$
\theta_{V, W}: F(V \otimes W) \rightarrow F(V) \otimes F(W), 1 \otimes 1 \mapsto \mu((i, j),(k, l)) 1 \otimes 1 .
$$

The associativity constraints yield that $\mu:\left(C_{p} \times C_{p}\right)^{2} \rightarrow K^{\times}$is a 2-cocycle. We shall compute $\mu$ explicitly in the sequel. We will see that:

$$
\theta_{K^{i, j}, K^{k, l}}: F\left(K^{i, j} \otimes K^{k, l}\right) \rightarrow F\left(K^{i, j}\right) \otimes F\left(K^{k, l}\right), 1 \otimes 1 \mapsto \zeta^{i l-j k} 1 \otimes 1 .
$$

3.4.3. One representation of $A_{0}$ and one representation of $A_{1}$. We next consider the case $V=K^{i, j}$ and $W=M$ (and $V=M$ and $W=K^{i, j}$ ). We first deal with the values $(i, j)=(0,1),(1,0)$ and then we will deduce a formula for an arbitrary pair $(i, j)$.

We need to find an isomorphism between $F\left(K^{1,0} \otimes M\right)$ and $F\left(K^{1,0}\right) \otimes F(M)$. Both representations are isomorphic to $M$. Thus, up to a nonzero scalar, there is only one possible choice. Using (3.3), we see that such an isomorphism must be given by

$$
\theta_{K^{1,0}, M}: F\left(K^{1,0} \otimes M\right) \rightarrow F\left(K^{1,0}\right) \otimes F(M), 1 \otimes m \mapsto \alpha_{1,0} \otimes\left(v_{a} v_{b}\right) m,
$$

for some $\alpha_{1,0} \in K$ (that will be determined later). In a similar fashion:

$$
\begin{array}{ll}
\theta_{K^{0,1}, M}: F\left(K^{0,1} \otimes M\right) \rightarrow F\left(K^{0,1}\right) \otimes F(M), & 1 \otimes m \mapsto \alpha_{0,1} \otimes\left(v_{b}^{-1} v_{a}^{-1}\right) m, \\
\theta_{M, K^{1,0}}: F\left(M \otimes K^{1,0}\right) \rightarrow F(M) \otimes F\left(K^{1,0}\right), & m \otimes 1 \mapsto \beta_{1,0}\left(v_{a}^{-1} v_{b}\right) m \otimes 1, \\
\theta_{M, K^{0,1}}: F\left(M \otimes K^{0,1}\right) \rightarrow F(M) \otimes F\left(K^{0,1}\right), & m \otimes 1 \mapsto \beta_{0,1}\left(v_{b}^{-1} v_{a}\right) m \otimes 1,
\end{array}
$$

for $\alpha_{0,1}, \beta_{1,0}, \beta_{0,1} \in K$.

The tensor structure on $F$ will depend on $\alpha_{1,0}, \alpha_{0,1}, \beta_{1,0}, \beta_{0,1}$, and $\mu$. The compatibility of $F$ with associativity constraints will impose some restrictions on the possible values of them.

We show by induction that the following formula holds for $(i, 0)$ with $i \geq 2$ :

$$
\theta_{K^{i, 0}, M}: F\left(K^{i, 0} \otimes M\right) \rightarrow F\left(K^{i, 0}\right) \otimes F(M), 1 \otimes m \mapsto \alpha_{1,0}^{i} \otimes\left(v_{a}^{i} v_{b}^{i}\right) m .
$$


Using naturality and compatibility of $F$ with the associativity constraint we have the following commutative diagram:

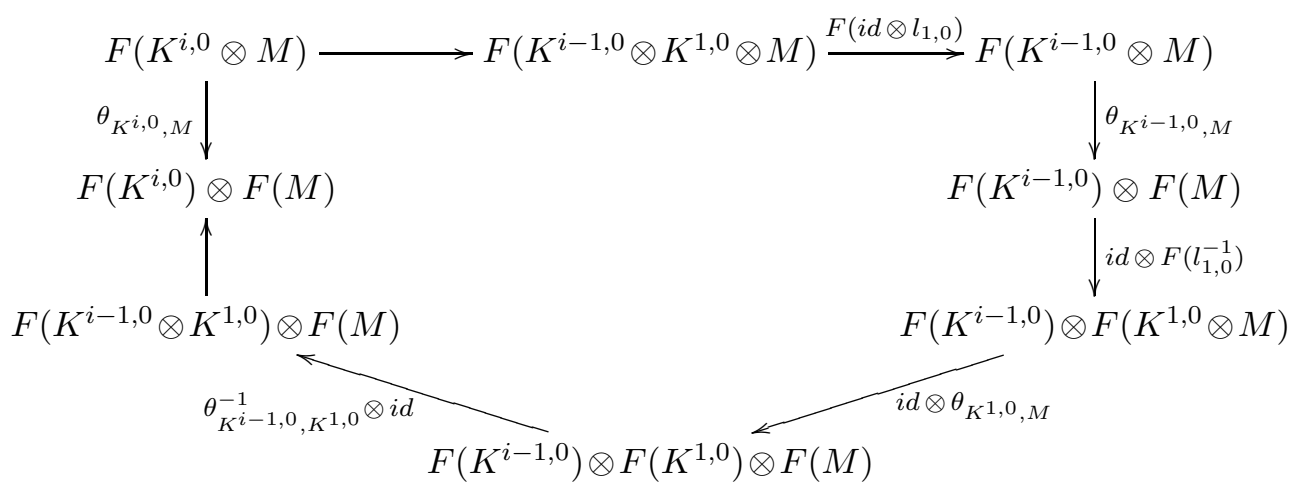

One can check that $1 \otimes m$ is mapped to

$$
\alpha_{1,0}^{i} \mu((i-1,0),(1,0))^{-1} \otimes\left(v_{a}^{i} v_{b}^{i}\right) m .
$$

Without loss of generality, we can assume that $\mu((i, 0),(j, 0))=\mu((0, i),(0, j))=1$, and then we arrive at the desired formula.

By a similar calculation we also obtain:

$$
\theta_{K^{0, j}, M}: F\left(K^{0, j} \otimes M\right) \rightarrow F\left(K^{0, j}\right) \otimes F(M), \quad 1 \otimes m \mapsto \alpha_{0,1}^{j} \otimes\left(v_{b}^{-j} v_{a}^{-j}\right) m .
$$

We can combine these two isomorphisms with the associativity constraint to get the following general formula:

$$
\theta_{K^{i, j}, M}: F\left(K^{i, j} \otimes M\right) \rightarrow F\left(K^{i, j}\right) \otimes F(M), 1 \otimes m \mapsto \alpha_{1,0}^{i} \alpha_{0,1}^{j} \zeta^{i(i-j)} \otimes v_{b}^{i-j} v_{a}^{i-j} m .
$$

This is done as follows. Using naturality and compatibility of $F$ with the associativity constraint we can construct the following commutative diagram:

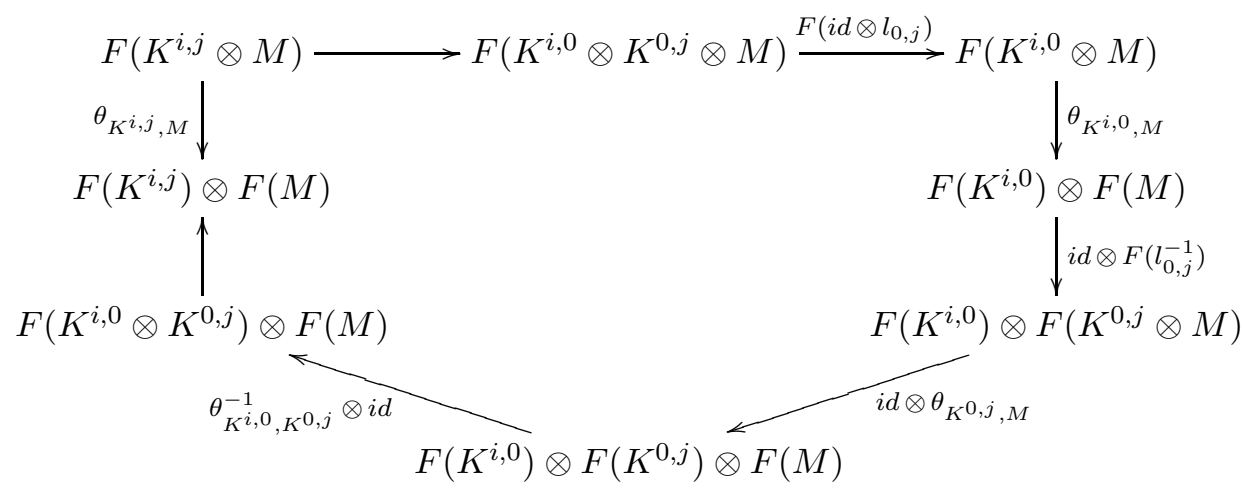

Following the longest path, we obtain:

$$
\theta_{K^{i, j}, M}(1 \otimes m)=\frac{\alpha_{1,0}^{i} \alpha_{0,1}^{j} \zeta^{i^{2}}}{\mu((i, 0),(0, j))} \otimes v_{b}^{i-j} v_{a}^{i-j} m
$$


We can write a similar diagram with $K^{0, j} \otimes K^{i, 0} \otimes M$ in the upper central term and proceeding accordingly we get:

$$
\theta_{K^{i, j}, M}(1 \otimes m)=\frac{\alpha_{1,0}^{i} \alpha_{0,1}^{j} \zeta^{i^{2}-2 i j}}{\mu((0, j),(i, 0))} \otimes v_{b}^{i-j} v_{a}^{i-j} m .
$$

These two equalities yield the following formula for $\mu$ :

$$
\frac{\mu((0, j),(i, 0))}{\mu((i, 0),(0, j))}=\zeta^{-2 i j}
$$

Since $C_{p} \times C_{p}$ is abelian and $K$ is assumed to be algebraically closed of characteristic zero, this completely determines the cohomology class of $\mu$. We choose the following representative from this cohomology class:

$$
\mu((i, j),(k, l))=\zeta^{i l-j k} .
$$

Substituting this in (3.7) we arrive at the desired formula for $\theta_{K^{i, j}, M}$. By making this choice we also assure that $F^{2}=I d$ on the subcategory of representations of $A_{0}$.

By a similar calculation, we obtain:

$$
\theta_{M, K^{i, j}}: F\left(M \otimes K^{i, j}\right) \rightarrow F(M) \otimes F\left(K^{i, j}\right), m \otimes 1 \mapsto \beta_{1,0}^{i} \beta_{0,1}^{j} \zeta^{j(j-i)} v_{a}^{j-i} v_{b}^{i-j} m \otimes 1 .
$$

We have described so the tensor structure on $F$ for the tensor product of representations of $A_{0}$ with representations of $A_{1}$. One can verify that this structure is indeed compatible with all the associativity constraints involving two irreducible representations of $A_{0}$ if and only if $\alpha_{1,0}, \alpha_{0,1}, \beta_{1,0}$, and $\beta_{0,1}$ are $p$-th roots of unity. Moreover, $F^{2}=I d$ on $K^{i, j} \otimes M$ and $M \otimes K^{i, j}$ if and only if

$$
\alpha_{1,0} \alpha_{0,1}=\beta_{1,0} \beta_{0,1}=1 \text {. }
$$

We shall assume that this holds henceforth.

3.4.4. Two representations of $A_{1}$. Lastly, we compute the isomorphism between $F(M \otimes M)$ and $F(M) \otimes F(M)$. We know that $M \otimes M \simeq \oplus_{i, j=0}^{p-1} K^{i, j}$. One can easily check that the element $q_{i, j} \in M \otimes M$ spanning the 1-dimensional representation isomorphic to $K^{i, j}$ must be of the form

$$
q_{i, j}=\lambda_{i, j} \sum_{t} \zeta^{-t j} m_{t} \otimes m_{i-t}, \text { with } \lambda_{i, j} \in K .
$$

(Unless otherwise specified, throughout the limits in the sums are understood to run from 0 to $p-1$.) We take $\lambda_{i, j}=1$ for every $i, j$. The isomorphism is given by:

$$
\theta_{M, M}: F(M \otimes M) \rightarrow F(M) \otimes F(M), q_{i, j} \mapsto \gamma_{i, j} q_{j, i},
$$


for some $\gamma_{i, j} \in K$. Using naturality and compatibility of $F$ with the associativity constraint at $K^{i, j} \otimes M \otimes M$ we obtain the following commutative diagram:

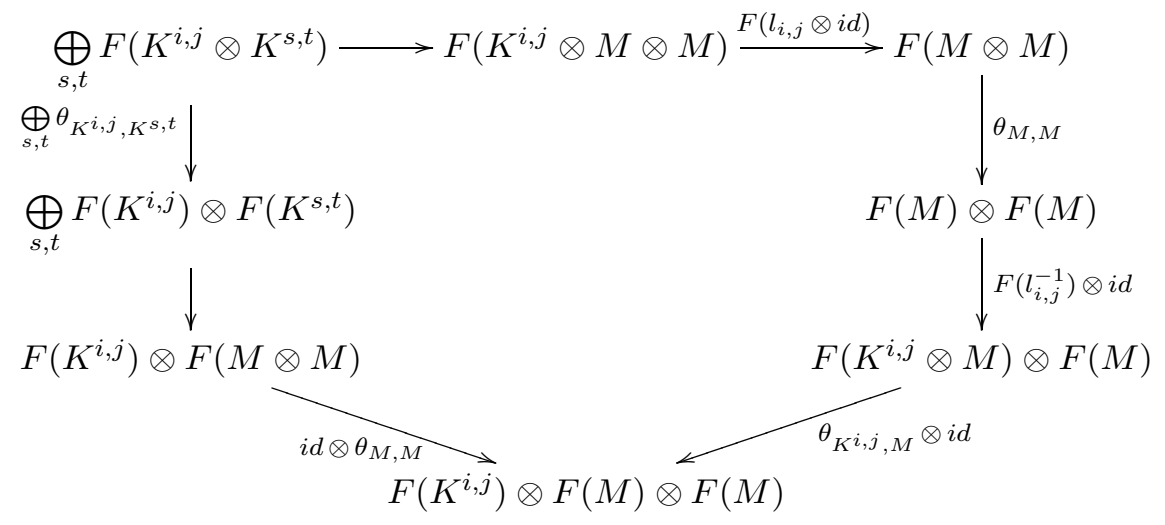

Through the isomorphism on the upper right side, $1 \otimes 1 \in K^{i, j} \otimes K^{s, t}$ is mapped to $\gamma_{s+i, t+j} \alpha_{1,0}^{i} \alpha_{0,1}^{j} \zeta^{i t-j s} \otimes q_{t, s}$. Through the isomorphism on the left side, $1 \otimes 1$ is mapped to $\gamma_{s, t} \zeta^{i t-j s} \otimes q_{t, s}$. From here,

$$
\gamma_{i, j}=\alpha_{1,0}^{j-i} \gamma_{0,0}
$$

By considering the associativity constraint for $M \otimes M \otimes K^{i, j}$ and writing the analogous diagram we get $\gamma_{i, j}=\beta_{1,0}^{j-i} \gamma_{0,0}$. This implies

$$
\alpha_{1,0}=\beta_{1,0} .
$$

The tensor structure of $F$ on $M \otimes M$ depends therefore on $\alpha_{1,0}$ (which is a $p$-th root of unity) and $\gamma_{0,0}$ (which equals \pm 1 since $F^{2}=I d$ on $M \otimes M$ ).

By checking compatibility with all associativity constraints we see that the isomorphism we have constructed does furnish a tensor structure on $F$. It can be shown directly that no matter what choice we make for $\gamma_{0,0}$ and $\alpha_{1,0}$, we will always end up with an isomorphic functor. We can thus assume, without loss of generality, that

$$
\gamma_{0,0}=\alpha_{1,0}=1 \text {. }
$$

Then, the scalars $\alpha_{0,1}, \beta_{1,0}, \beta_{0,1}$, and $\gamma_{i, j}$ equal 1 by equations (3.9), (3.11), and (3.12). This finishes the description of the tensor structure on $F$.

We summarize our discussion in the following result.

Proposition 3.2. Let $A$ be the Hopf algebra defined in Subsection 3.1. Consider its irreducible representations $K^{i, j}$, with $0 \leq i, j<p$, and $M$ defined in Subsection 3.3 . There exists (up to isomorphism) only one tensor functor $F: \operatorname{Rep}(A) \rightarrow \operatorname{Rep}(A)$ such that $F\left(K^{i, j}\right) \simeq K^{j, i}$ and $F(M) \simeq M$. It is given by the equations (3.5), (3.6), (3.8), and (3.10), where the scalars $\alpha_{1,0}, \alpha_{0,1}, \beta_{1,0}, \beta_{0,1}$, and $\gamma_{i, j}$ equal 1 .

3.5. The comultiplication of $g$. The category $\operatorname{Rep}(H)$ can be identified with that of $F$-equivariant representations of $A$ as follows: if $V \in \operatorname{Rep}(H)$, then $V \in \operatorname{Rep}(A)$ by restriction, and $\tilde{g}: V \rightarrow V, v \mapsto g v$ establishes an isomorphism between $V$ and $F(V)$. 
We now consider the regular representation of $H$. The following diagram should be commutative:

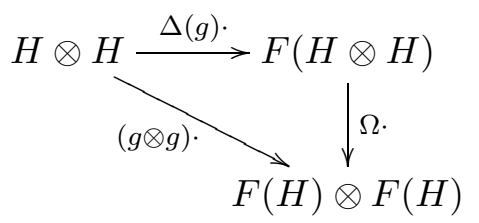

where $\Omega$ comes from the tensor structure of $F$. Since $g=g^{-1}$, we have:

$$
\Delta(g)=(g \otimes g) \Omega .
$$

For $V, W \in \operatorname{Rep}(A)$ the isomorphism $\theta_{V, W}: F(V \otimes W) \rightarrow F(V) \otimes F(W)$ is given by multiplication by $\Omega \in A \otimes A$. The reason for this is the following: the isomorphism $\theta_{A, A}: A \otimes A \simeq F(A \otimes A) \rightarrow F(A) \otimes F(A) \simeq A \otimes A$ is natural, and hence it must commute with multiplication from the right by elements of $A \otimes A$. So, it must be given by multiplication from the left by some element $\Omega \in A \otimes A$. The same holds for $V, W \in \operatorname{Rep}(A)$ by the naturality of $\theta$ again with respect to any morphisms $A \rightarrow V$ and $A \rightarrow W$. Then, the computation of $\Omega$ can be derived from our knowledge of these isomorphisms for any two irreducible representations of $A$. To do this, we first need the decomposition of the regular representation of $A$ as a direct sum of irreducible representations. For $i, j=0, \ldots, p-1$ let $f_{i j} \in A_{0}$ denote the idempotent upon which $u_{a}$ acts by $\zeta^{i}$ and $u_{b}$ by $\zeta^{j}$. It is:

$$
f_{i j}=\frac{1}{p^{2}} \sum_{k, l} \zeta^{-(i k+j l)} u_{a}^{k} u_{b}^{l} .
$$

Let $V_{i j}=A_{0} f_{i j}$. Then $V_{i j} \simeq K^{i, j}$. Consider in $A_{1}$ the element

$$
h_{i}=\frac{1}{p} \sum_{k} \zeta^{-i k} v_{a}^{k}
$$

Let $W_{i}$ be the subspace spanned by $v_{b}^{l} h_{i}$ for $l=0, \ldots, p-1$. Then $W_{i} \simeq M$ by mapping $v_{b}^{l-i} h_{i}$ to $m_{l}$. Thus we have:

$$
A=\left(\bigoplus_{i, j} V_{i j}\right) \bigoplus\left(\bigoplus_{i} W_{i}\right) .
$$

We claim that:

$$
\begin{aligned}
\Omega= & \frac{1}{p^{2}} \sum_{i, j, k, l} \zeta^{k j-i l} u_{a}^{i} u_{b}^{j} \otimes u_{a}^{k} u_{b}^{l}+\frac{1}{p} \sum_{k, l} \zeta^{-(k+l) k} u_{a}^{k} u_{b}^{l} \otimes v_{a}^{k+l} v_{b}^{k+l} \\
& +\frac{1}{p} \sum_{k, l} \zeta^{k(k+l)} v_{a}^{k+l} v_{b}^{-(k+l)} \otimes u_{a}^{k} u_{b}^{l}+\frac{1}{p} \sum_{k, l} v_{a}^{k} v_{b}^{l} \otimes v_{a}^{-l} v_{b}^{k} .
\end{aligned}
$$

Using (3.5), (3.8), (3.6), and (3.10), this formula for $\Omega$ is proved by checking directly the following equalities, which we leave to the reader:

$$
\begin{aligned}
& \theta_{V_{i j}, V_{k l}}\left(f_{i j} \otimes f_{k l}\right)=\zeta^{i l-j k} f_{i j} \otimes f_{k l}=\Omega\left(f_{i j} \otimes f_{k l}\right), \\
& \theta_{V_{i j}, M}\left(f_{i j} \otimes v_{b}^{k-l} h_{l}\right)=\zeta^{(i-j)(k+i)} f_{i j} \otimes v_{b}^{k+i-j-l} h_{l}=\Omega\left(f_{i j} \otimes v_{b}^{k-l} h_{l}\right), \\
& \theta_{M, V_{i j}}\left(v_{b}^{k-l} h_{l} \otimes f_{i j}\right)=\zeta^{(j-i)(k+i)} v_{b}^{k+i-j-l} h_{l} \otimes f_{i j}=\Omega\left(v_{b}^{k-l} h_{l} \otimes f_{i j}\right),
\end{aligned}
$$




$$
\begin{aligned}
\theta_{M, M}\left(\sum_{k} \zeta^{-j k} v_{b}^{k-l} h_{l} \otimes v_{b}^{i-k-l} h_{l}\right)= & \sum_{k} \zeta^{-i k} v_{b}^{k-l} h_{l} \otimes v_{b}^{j-k-l} h_{l} \\
& =\Omega\left(\sum_{k} \zeta^{-j k} v_{b}^{k-l} h_{l} \otimes v_{b}^{i-k-l} h_{l}\right) .
\end{aligned}
$$

A careful calculation reveals that $S(g)=g$. This finishes the description of the Hopf algebra structure of $H$ and hence the proof of Theorem 3.1 .

Remark 3.3. Although we used that $K$ is algebraically closed to reconstruct $H$, a posteriori we see from Theorem 3.1 that $H$ is defined over $\mathbb{Q}(\zeta)$.

\section{Duality, (CO)Characters, and Hopf automorphisms}

In this section we study further the structure of $H$ : we describe its irreducible (co)representations and (co)characters, its Hopf automorphisms and we show that it is self-dual. The description of the (co)characters is one of the essential points in the proof of our main result since they provide elements in any Hopf order in view of Proposition 1.2. We keep the notation of the previous section.

4.1. Dual Hopf algebra. We present here the Hopf algebra structure of $H^{*}$. As a vector space, $H=A_{0} \oplus A_{1} \oplus g A_{0} \oplus g A_{1}$. We consider the following basis of $H$ :

$$
\mathcal{B}:=\left\{u_{a}^{i} u_{b}^{j}\right\} \cup\left\{v_{a}^{i} v_{b}^{j}\right\} \cup\left\{g u_{a}^{i} u_{b}^{j}\right\} \cup\left\{g v_{a}^{i} v_{b}^{j}\right\} .
$$

We denote the dual basis by:

$$
\mathcal{B}^{*}:=\left\{s_{i j}\right\} \cup\left\{t_{i j}\right\} \cup\left\{\alpha_{i j}\right\} \cup\left\{\beta_{i j}\right\} .
$$

From (3.1) and (3.2), we easily see that $H=A \oplus g A$ as a coalgebra. Then

$$
H^{*}=A^{*} \oplus(g A)^{*}
$$

as an algebra. We denote by $\varepsilon_{A}$ and $\varepsilon_{g A}$ the counit of $H$ restricted to $A$ and $g A$ respectively. Then, $\varepsilon_{A}$ and $\varepsilon_{g A}$ are the central idempotents of $H^{*}$ giving the previous decomposition. The following result provides the full description of $H^{*}$.

Proposition 4.1. As an algebra, $H^{*}$ is the direct sum of the algebras $A^{*}$ and $(g A)^{*}$. The algebra $A^{*}$ is spanned by the elements $s_{i j}$ and $t_{i j}$ and its multiplication is given by:

$$
\begin{array}{ll}
s_{i j} s_{k l}=\delta_{i, k} \delta_{j, l} s_{i j}, & t_{k l} s_{i j}=\delta_{i, k} \delta_{j,-l} t_{k l}, \\
s_{i j} t_{k l}=\delta_{i, k} \delta_{j, l} t_{k l}, & t_{i j} t_{k l}=\delta_{i, k} \delta_{j,-l} s_{i j} .
\end{array}
$$

The algebra $(g A)^{*}$ is generated by the elements $\gamma_{i j}$ and $B$ subject to the following relations:

$$
B^{2}=\varepsilon_{g A}, \quad \gamma_{i j} \gamma_{k l}=\zeta^{i l-j k} \gamma_{i+k j+l}, \quad \text { and } \quad B \gamma_{i j}=\gamma_{i j} B .
$$


The comultiplication, counit, and antipode of $H^{*}$ are given by:

$$
\begin{aligned}
& \Delta\left(s_{i j}\right)=\sum_{k, l} s_{k l} \otimes s_{i-k j-l}+\frac{1}{p^{2}} \zeta^{-(i l+j k)} \gamma_{k l} \otimes \gamma_{l k}, \\
& \varepsilon\left(s_{i j}\right)=\delta_{i, 0} \delta_{j, 0}, \quad S\left(s_{i j}\right)=s_{-i-j}, \\
& \Delta\left(t_{i j}\right)=\sum_{k, l} \zeta^{l(k-i)} t_{k l} \otimes t_{i-k j-l}+\frac{1}{p^{2}} \zeta^{-i l} \gamma_{k l} B \otimes \gamma_{l-j k} B, \\
& \varepsilon\left(t_{i j}\right)=\delta_{i, 0} \delta_{j, 0}, \quad S\left(t_{i j}\right)=\zeta^{-i j} t_{-i j}, \\
& \Delta\left(\gamma_{i j}\right)=\sum_{k, l} \zeta^{l i+k j} s_{k l} \otimes \gamma_{i j}+\zeta^{k i+l j} \gamma_{i j} \otimes s_{k l}, \\
& \varepsilon\left(\gamma_{i j}\right)=0, \quad S\left(\gamma_{i j}\right)=\gamma_{-j-i}, \\
& \Delta(B)=\sum_{k, l} \zeta^{k l} \gamma_{l 0} B \otimes t_{k l}+t_{k l} \otimes \gamma_{0-l} B, \\
& \varepsilon(B)=0, \quad S(B)=B .
\end{aligned}
$$

(The operations in the indices are all done modulo $p$.)

Proof. From the dual basis $\mathcal{B}^{*}$ in (4.2), we are going to construct a new basis of $H^{*}$ which is more convenient to express the multiplication. In $\left(g A_{0}\right)^{*}$, instead of $\left\{\alpha_{i j}\right\}$ we take the dual basis of $\left\{g f_{i j}\right\}$, where $\left\{f_{i j}\right\}$ are the idempotents in (3.13). We denote this basis by $\left\{\gamma_{i j}\right\}$. Then:

$$
\gamma_{i j}\left(g u_{a}^{k} u_{b}^{l}\right)=\zeta^{i k+j l}
$$

The $s_{i j}$ 's and $t_{i j}$ 's form a basis of $A^{*}$ and the $\beta_{i j}$ 's and $\gamma_{i j}$ 's form one of $(g A)^{*}$. A direct and tedious calculation yields the following formulas:

$$
\begin{array}{rlrl}
s_{i j} s_{k l} & =\delta_{i, k} \delta_{j, l} s_{i j}, & t_{k l} s_{i j}=\delta_{i, k} \delta_{j,-l} t_{k l}, \\
s_{i j} t_{k l}=\delta_{i, k} \delta_{j, l} t_{k l}, & t_{i j} t_{k l}=\delta_{i, k} \delta_{j,-l} s_{i j}, \\
\gamma_{i j} \beta_{k l}=\zeta^{j(l+j+k-i)} \beta_{k-i+j l-i+j}, & \beta_{k l} \gamma_{i j}=\zeta^{j(i+k-j-l)} \beta_{k+i-j l+j-i}, \\
\gamma_{i j} \gamma_{k l}=\zeta^{i l-j k} \gamma_{i+k j+l} . &
\end{array}
$$

This gives the statement for the multiplication in $A^{*}$. For the one in $(g A)^{*}$ we proceed as follows: consider the element

$$
B=\sqrt{p} \sum_{k} \beta_{k 0} .
$$

It commutes with the $\gamma_{i j}$ 's in view of the above formulas. A simple computation shows that $B^{2}=\varepsilon_{g A}$. Each $\beta_{i j}$ can be expressed as

$$
\beta_{i j}=\frac{1}{p \sqrt{p}} \sum_{k} \zeta^{-k i} \gamma_{k-j k} B .
$$

This can be verified directly by using the equality:

$$
\left(\gamma_{i j} B\right)\left(g v_{a}^{k} v_{b}^{l}\right)=\sqrt{p} \zeta^{j k} \delta_{l, j-i} .
$$


Then $\left\{\gamma_{i j}\right\} \cup\left\{\gamma_{i j} B\right\}$ is a basis of $(g A)^{*}$. We change our basis of $H^{*}$ again to

$$
\mathcal{L}:=\left\{s_{i j}\right\} \cup\left\{t_{i j}\right\} \cup\left\{\gamma_{i j}\right\} \cup\left\{\gamma_{i j} B\right\} .
$$

The multiplication of $H^{*}$ is then fully described on $\mathcal{L}$ by (4.4) and (4.5).

We next compute the formulas for the comultiplication of $H^{*}$ given in (4.6). These formulas follow from direct calculations, just using the multiplication in $H$. The calculations do not present any special difficulty. We briefly indicate how to proceed for $s_{i j}$ and leave the details and the other cases to the reader. The element $s_{i j}$ vanishes on $A_{1}, g A_{0}$ and $g A_{1}$. Since $A_{0} A_{0}=\left(g A_{0}\right)\left(g A_{0}\right)=A_{0}$ and $A_{0} A_{1}=A_{1} A_{0}=$ 0 no other kind of summands can occur in the right-hand side. Hence it suffices to evaluate $\Delta\left(s_{i j}\right)$ at $u_{a}^{k} u_{b}^{l} \otimes u_{a}^{m} u_{b}^{n}$ and $g f_{k l} \otimes g f_{m n}$. The coefficients of $s_{k l} \otimes s_{m n}$ and $\gamma_{k l} \otimes \gamma_{m n}$ must be respectively:

$$
\left\langle s_{i j},\left(u_{a}^{k} u_{b}^{l}\right)\left(u_{a}^{m} u_{b}^{n}\right)\right\rangle=\delta_{i, k+m} \delta_{j, l+n} \quad \text { and } \quad\left\langle s_{i j},\left(g f_{k l}\right)\left(g f_{m n}\right)\right\rangle=\frac{1}{p^{2}} \zeta^{-(i l+j k)} \delta_{k, n} \delta_{l, m} .
$$

Finally, one can check with no effort that the counit and antipode are the ones given in (4.6).

4.2. Self-duality. Nikshych proved in [11, Proposition 5.2] that $H$ and $H^{*}$ are isomorphic as algebras. In this subsection we strengthen this result by the following proposition:

Proposition 4.2. The Hopf algebras $H$ and $H^{*}$ are isomorphic.

Proof. Let us begin by finding inside $H^{*}$ a Hopf subalgebra isomorphic to $A$. Set $d=\frac{p+1}{2}$. Consider the elements:

$$
\bar{u}_{a}=\sum_{k, l} \zeta^{(k+l) d} s_{k l}, \quad \bar{u}_{b}=\sum_{k, l} \zeta^{(k-l) d} s_{k l}, \quad \bar{v}_{a}=\gamma_{d d}, \quad \bar{v}_{b}=\gamma_{-d d}
$$

Let $\bar{A}$ be the subalgebra generated by $\bar{u}_{a}, \bar{u}_{b}, \bar{v}_{a}$, and $\bar{v}_{b}$. Using the multiplication rules (4.4) and (4.5) one easily checks that the assignment $u_{x} \mapsto \bar{u}_{x}, v_{x} \mapsto \bar{v}_{x}$ for $x \in\{a, b\}$ establishes an algebra isomorphism $\Psi$ between $A$ and $\bar{A}$. The elements corresponding to the central idempotents $e_{0}$ and $e_{1}$ in Subsection 3.1 are

$$
\varepsilon_{A}=\sum_{k, l} s_{k l} \quad \text { and } \quad \varepsilon_{g A}=\gamma_{00} .
$$

Notice that $\varepsilon_{A}+\varepsilon_{g A}=\varepsilon_{H}=1_{H^{*}}$. Using formulas (4.6) one can verify with a long but direct computation that the above isomorphism is actually an isomorphism of Hopf algebras.

Consider finally the element

$$
\bar{g}=B+\sum_{k, l} \zeta^{d k l} t_{k l}
$$

It can be shown that $\bar{g}^{2}=1_{H^{*}}$, conjugation by $\bar{g}$ stabilizes $\bar{A}$, and, by the above isomorphism, $\bar{g}$ acts on $\bar{A}$ as $g$ acts on $A$. Moreover, one can show that $\Psi$ extends to a Hopf algebra isomorphism from $H$ to $H^{*}$ by defining $g \mapsto \bar{g}$. This finishes the proof. 
Remark 4.3. If $p=1 \bmod$. 4 , then $\sqrt{p} \in \mathbb{Q}(\zeta)$ and the above isomorphism is defined over $\mathbb{Q}(\zeta)$. Otherwise, it is not defined over $\mathbb{Q}(\zeta)$ but over $\mathbb{Q}(\zeta, \omega)$, with $\omega$ a primitive fourth root of unity, and maps $B$ to $\omega B$. Consider $H$ as defined over $\mathbb{Q}(\zeta)$. Then $B$ belongs to $H \otimes_{\mathbb{Q}(\zeta)} K$ but not to $H$ because $\sqrt{p} \notin \mathbb{Q}(\zeta)$ in this case. In fact, since the orbit of $B$ under the group of Hopf automorphisms of $H$ is $\{B,-B\}$, see Subsection 4.5, it follows that an isomorphism between $H$ and $H^{*}$ cannot be defined over $\mathbb{Q}(\zeta)$. The Hopf algebra $H^{*}$ will be a form of $H$ but not isomorphic to it over $\mathbb{Q}(\zeta)$.

In the next two subsections we describe the irreducible representations of $H$ and $H^{*}$ and their characters, see [11, Proposition 5.2], which will be used to find the possible Hopf orders of $H$.

4.3. Characters of $H$. We have the following irreducible representations of $H$ and corresponding characters:

4.3.1. Dimension 1. There are $2 p$ irreducible representations of $H$ of dimension 1 . They arise from the elements in $A_{0}$ that are $g$-invariant. For $i=0, \ldots, p-1$ we have the representation $V_{i}^{+}$(resp. $V_{i}^{-}$), upon which $A_{1}$ acts trivially, $u_{a}^{k} u_{b}^{l}$ acts through the scalar $\zeta^{(k+l) i}$, and $g$ acts as 1 (resp. -1). By using the previously chosen basis $\mathcal{L}$ of $H^{*}$ (see Equation 4.9) we can write the characters of these representations as:

$$
\chi_{V_{i}^{ \pm}}= \pm \gamma_{i i}+\sum_{k, l} \zeta^{(k+l) i} s_{k l}
$$

4.3.2. Dimension 2. The irreducible representations of $H$ of dimension 2 come from the 1-dimensional representations of $A_{0}$ which are not $g$-invariant. Therefore, their orbits have two elements: $K^{i, j}$ and $K^{j, i}$ for $i \neq j$. Such representations are parameterized by pairs $(i, j)$ with $i<j$. We denote them by $W_{i j}$. There are $\frac{p(p-1)}{2}$ such representations. The elements $g$ and $u_{a}^{k} u_{b}^{l}$ act on $W_{i j}$ as the matrices

$$
\left(\begin{array}{ll}
0 & 1 \\
1 & 0
\end{array}\right) \text { and }\left(\begin{array}{cc}
\zeta^{i k+j l} & 0 \\
0 & \zeta^{i l+j k}
\end{array}\right)
$$

respectively, and $A_{1}$ acts trivially. The associated characters with respect to the basis $\mathcal{L}$ of $H^{*}$ are:

$$
\chi_{W_{i j}}=\sum_{k, l}\left(\zeta^{i k+j l}+\zeta^{i l+j k}\right) s_{k l} .
$$

4.3.3. Dimension $p$. Finally, there are two irreducible representations of $H$ of dimension $p$. They arise from the $p$-dimensional representation $M$ of $A_{1}$, see Subsection 3.3. We denote them by $M^{+}$and $M^{-}$. They have basis $\left\{m_{0}, \ldots, m_{p-1}\right\}$, the elements in $A_{1}$ act as $v_{a} m_{i}=\zeta^{i} m_{i}, v_{b} m_{i}=m_{i+1}$ and $g$ acts as \pm 1 . The elements of $A_{0}$ act trivially. The corresponding characters in the basis $\mathcal{L}$ of $H^{*}$ are:

$$
\chi_{M^{ \pm}}=p t_{00} \pm \frac{1}{\sqrt{p}} \sum_{i} \gamma_{i i} B .
$$


4.4. Characters of $H^{*}$. To describe the irreducible representations of $H^{*}$ we will use the decomposition (4.3) expressing $H^{*}$ as the direct sum of algebras $H^{*}=A^{*} \oplus$ $(g A)^{*}$. We start with the irreducible representations of $A^{*}$. By the multiplication rules (4.4), $A^{*}$ is the direct sum of algebras

$$
A^{*}=\left(\bigoplus_{i} R_{i}\right) \bigoplus\left(\bigoplus_{i, j} R_{i, j}\right)
$$

where $R_{i}$ is spanned by $s_{i 0}$ and $t_{i 0}$ and $R_{i j}$ by $s_{i j}, s_{i-j}, t_{i j}, t_{i-j}$. The index $i$ runs from 0 to $p-1$ and $j$ from 1 to $\frac{p-1}{2}$ to avoid repetitions.

4.4.1. Dimension 1. The algebra $R_{i}$ has two 1-dimensional representations, on both of which $s_{i 0}$ acts as 1 whereas $t_{i 0}$ acts as \pm 1 . We denote them by $L_{i}^{+}$and $L_{i}^{-}$ respectively. The characters of these representations, expressed in the basis $\mathcal{B}$ of $H$, see Equation 4.1, are:

$$
\psi_{L_{i}^{ \pm}}=u_{a}^{i} \pm v_{a}^{i}
$$

4.4.2. Dimension 2. The algebra $R_{i j}$ is isomorphic to $\mathrm{M}_{2}(K)$. Therefore, it has one irreducible 2-dimensional representation, which we denote by $P_{i j}$. This representation is given by the following map:

$$
s_{i j} \mapsto\left(\begin{array}{cc}
1 & 0 \\
0 & 0
\end{array}\right), \quad s_{i-j} \mapsto\left(\begin{array}{cc}
0 & 0 \\
0 & 1
\end{array}\right), \quad t_{i j} \mapsto\left(\begin{array}{cc}
0 & 1 \\
0 & 0
\end{array}\right), \quad t_{i-j} \mapsto\left(\begin{array}{cc}
0 & 0 \\
1 & 0
\end{array}\right) .
$$

In the basis $\mathcal{B}$ of $H$ the characters of these representations are expressed as:

$$
\psi_{P_{i j}}=u_{a}^{i} u_{b}^{j}+u_{a}^{i} u_{b}^{-j}
$$

4.4.3. Dimension $p$. Lastly, we discuss the irreducible representations of $(g A)^{*}$. Since $B^{2}=\left.\varepsilon\right|_{g A}=1_{(g A)^{*}}$, we have the following two central idempotents:

$$
\kappa=\frac{1}{2}\left(\left.\varepsilon\right|_{g A}+B\right) \quad \text { and } \quad \kappa^{\prime}=\frac{1}{2}\left(\left.\varepsilon\right|_{g A}-B\right) .
$$

They induce the algebra decomposition $(g A)^{*}=(g A)^{*} \kappa \oplus(g A)^{*} \kappa^{\prime}$. From (4.5) we obtain $\gamma_{10}^{p}=\gamma_{01}^{p}=\varepsilon_{g A}$ and $\gamma_{10} \gamma_{01}=\zeta^{2} \gamma_{01} \gamma_{10}$. Then $(g A)^{*} \kappa$ and $(g A)^{*} \kappa^{\prime}$ are isomorphic to $\mathrm{M}_{p}(K)$. Hence $(g A)^{*}$ has two $p$-dimensional irreducible representations, which we denote by $N^{+}$and $N^{-}$. Both have a basis $\left\{n_{0}, \ldots, n_{p-1}\right\}$ with actions

$$
\gamma_{i j} n_{l}=\zeta^{i j+2 i l} n_{l+j}, \quad B n_{l}= \pm n_{l} .
$$

The characters of the above representations are given by:

$$
\psi_{N^{ \pm}}=\frac{1}{p} \sum_{i, j} g u_{a}^{i} u_{b}^{j} \pm \frac{1}{\sqrt{p}} \sum_{i} g v_{a}^{i}
$$


4.5. Hopf automorphisms. The group of Hopf automorphisms of $H$ is described by the following result:

Proposition 4.4. The group Aut $\mathrm{t}_{\text {Hopf }}(H)$ is isomorphic to $C_{2} \times\left(C_{2} \ltimes C_{p}\right)$. Writing $C_{2}$ as $\{ \pm 1\}$, the Hopf automorphism $\phi$ of $H$ corresponding to the triple $\left(\epsilon_{1}, \epsilon_{2}, t\right)$ is:

$$
\begin{array}{lll}
\phi\left(u_{a}\right)=u_{a}^{\epsilon_{2}}, & \phi\left(u_{b}\right)=u_{b}^{\epsilon_{2}}, & \\
\phi\left(v_{a}\right)=v_{a}^{\epsilon_{2}}, & \phi\left(v_{b}\right)=\zeta^{t} v_{b}^{\epsilon_{2}}, & \phi(g)=g\left(e_{0}+\epsilon_{1} e_{1}\right) .
\end{array}
$$

Proof. We know from (3.1) and (3.2) that $H=A \oplus g A$ as coalgebras and hence $H^{*}=A^{*} \oplus(g A)^{*}$ as algebras. The algebra $A^{*}$ splits as a direct sum of matrix algebras over $K$ of dimension 1 or 4 (Subsections 4.4.1 and 4.4.2). On the other hand, the algebra $(g A)^{*}$ is the direct sum of two matrix algebras of dimension $p^{2}$ (Subsection 4.4.3). Let $\sigma \in A u t_{H o p f}(H)$. Since $\sigma$ must preserve the Wedderburn decomposition of $H^{*}$, it must hold that $\sigma(A) \subseteq A$. Thus $\left.\sigma\right|_{A}$ is a Hopf automorphism of $A$. We are so led to compute $A u t_{\text {Hopf }}(A)$. This gives a group morphism

$$
\Theta: \operatorname{Aut}_{\text {Hopf }}(H) \rightarrow A u t_{\text {Hopf }}(A),\left.\sigma \mapsto \sigma\right|_{A} .
$$

Using this morphism, we are going to compute $A u t_{H o p f}(H)$ in two steps:

Step 1. Hopf automorphisms of $A$. We know from Subsection 3.1 that $A$ has an algebra decomposition $A=A_{0} \oplus A_{1}$, where $A_{0}=K\left(C_{p} \times C_{p}\right)$ and $A_{1}=K^{c}\left(C_{p} \times C_{p}\right)$. Considering, as before, the dimensions of the simple components of the Wedderburn decomposition of $A_{0}$ and $A_{1}$ we get $\sigma\left(A_{0}\right)=A_{0}$ and $\sigma\left(A_{1}\right)=A_{1}$. The group-like elements of $A$ are $u_{a}^{i} \pm v_{a}^{i}$ with $0 \leq i<p$. Since $\sigma$ preserves group-like elements and the relations $u_{a}^{p}=e_{0}$ and $v_{a}^{p}=e_{1}$, we must have $\sigma\left(u_{a}+v_{a}\right)=u_{a}^{r}+v_{a}^{r}$ for some $r \neq 0$. As $\sigma\left(u_{a}\right) \in A_{0}$ and $\sigma\left(v_{a}\right) \in A_{1}$, we obtain

$$
\sigma\left(u_{a}\right)=u_{a}^{r} \quad \text { and } \quad \sigma\left(v_{a}\right)=v_{a}^{r} .
$$

On the other hand, $\sigma\left(u_{b}\right)=u_{a}^{k} u_{b}^{s}$ for some $k, s \neq 0$ because $\sigma$ induces a Hopf automorphism on the quotient Hopf algebra $A_{0}$ of $A$. We derive that $k=0$ from the equality $\mu \Delta \sigma\left(u_{b}\right)=\sigma \mu \Delta\left(u_{b}\right)$. Here $\mu$ stands for the multiplication of $H$. So $\sigma\left(u_{b}\right)=u_{b}^{s}$. Using the equality $\Delta \sigma\left(u_{b}\right)=(\sigma \otimes \sigma) \Delta\left(u_{b}\right)$ we arrive to $\sigma\left(v_{b}\right)=\lambda v_{b}^{s}$ for some $\lambda \in K^{\times}$. Moreover, $\lambda^{p}=1$ because $\sigma\left(v_{b}\right)^{p}=e_{1}$. Put $\lambda=\zeta^{t}$ with $0 \leq t<p$. Applying $\sigma$ to the relation $v_{a} v_{b}=\zeta v_{b} v_{a}$ we get $s r=1 \bmod$. $p$. Then

$$
\sigma\left(u_{b}\right)=u_{b}^{s} \quad \text { and } \quad \sigma\left(v_{b}\right)=\zeta^{t} v_{b}^{s} \text {, with } s=r^{-1} \bmod . p .
$$

Thus $\sigma$ determines a pair $(r, t) \in C_{p}^{\times} \times C_{p}$. Conversely, one can check that any such a pair together with (4.18) and (4.19) defines a Hopf automorphism of $A$. Finally, by composing two automorphisms one sees that $A u t_{\text {Hopf }}(A) \simeq C_{p}^{\times} \ltimes C_{p}$.

Step 2. Computing the kernel and image of $\Theta$. We claim that $\operatorname{Ker} \Theta \simeq C_{2}$. Let $\nu \in \operatorname{Ker} \Theta$. We know that $H$ has a coalgebra decomposition $H=A \oplus g A$, that $\nu$ must preserve. Then $\nu(g)=g z$ for some $z \in A$. Since $\left.\nu\right|_{A}=i d_{A}$, we have for every $x \in A$ :

$$
g x g^{-1}=\nu\left(g x g^{-1}\right)=g z x z^{-1} g^{-1} .
$$


From this it follows that $z \in Z(A)$. Recall that $\Delta(g)=(g \otimes g) \Omega$, where $\Omega$ is given in Equation 3.14. Using this and that $\nu$ is a coalgebra map we get:

$$
(g \otimes g) \Omega \Delta(z)=\Delta(g z)=\Delta \nu(g)=(\nu \otimes \nu) \Delta(g)=(g z \otimes g z) \Omega .
$$

We also used here that $\Omega \in A \otimes A$ and $\left.\nu\right|_{A}=i d_{A}$. Since $z \in Z(A)$ and $\Omega$ and $g$ are invertible, the above equality implies that $z$ is a group-like element of $A$. As $1=\nu(g)^{2}=g z g z$, the only nontrivial option is $z=e_{0}-e_{1}$. Conversely, one can easily check that a map of this form defines an element of order 2 in $\operatorname{Ker} \Theta$.

We claim now that $\operatorname{Im} \Theta \simeq C_{2} \ltimes C_{p}$. Let $\sigma \in \operatorname{Im} \Theta$. Assume that $\sigma$ is given by $(r, t) \in C_{p}^{\times} \ltimes C_{p}$ and equations (4.18) and (4.19). Then, arguing as before, $\sigma(g)=g z$ for some $z \in A$. We have:

$$
u_{b}^{r^{-1}}=\sigma\left(u_{b}\right)=\sigma\left(g u_{a} g^{-1}\right)=g z u_{a}^{r} z^{-1} g^{-1}=u_{b}^{r} .
$$

From this, $r^{2}=1$ mod. $p$ and so $r= \pm 1$. Conversely, the Hopf automorphism $\tau$ of $A$ corresponding to $(1, t)$ is given by conjugation by the group-like element $u_{a}^{t}+v_{a}^{t}$. Conjugation by the same element defines $\bar{\tau} \in A u t_{H o p f}(H)$ such that $\Theta(\bar{\tau})=\tau$. Let $\varphi \in \operatorname{Aut}_{\text {Hopf }}(A)$ be corresponding to $(-1,0)$. One can check effortless that $\varphi \in \operatorname{Im} \Theta$ with preimage $\bar{\varphi}$ defined by $\left.\bar{\varphi}\right|_{A}=\varphi$ and $\bar{\varphi}(g)=g$.

Thus we have a short exact sequence

$$
1 \rightarrow C_{2} \rightarrow \operatorname{Aut}_{\text {Hopf }}(H) \rightarrow C_{2} \ltimes C_{p} \rightarrow 1 .
$$

This sequence splits because $\bar{\varphi}$ has order 2. The action on $C_{2}$ is trivial (this is the only possible action), and then

$$
\operatorname{Aut}_{\text {Hopf }}(H) \simeq C_{2} \times\left(C_{2} \ltimes C_{p}\right) .
$$

\section{Orders OF NikshyCh'S Hopf Algebra}

In this section we will use the results of the previous sections to classify the orders of Nikshych's Hopf algebra. We will see that Nikshych's Hopf algebra admits at most one order over any number field.

We keep the conventions and notations of Section $3, \zeta$ is a primitive $p$-th root of unity; $K$ is a number field containing $\zeta ; R=\mathcal{O}_{K}$ is the ring of integers of $K ; H$ denotes Nikshych's Hopf algebra of dimension $4 p^{2}$, and $A$ stands for Masuoka's Hopf algebra of dimension $2 p^{2}$, both defined over $K$.

Recall from Remark 3.3 that $H$ is defined over $\mathbb{Q}(\zeta)$. However, we will prove here that $H$ does not have orders over $\mathcal{O}_{\mathbb{Q}(\zeta)}$, but only over the ring of integers of some extension of $\mathbb{Q}(\zeta)$. Set $K=\mathbb{Q}(\zeta, \omega)$, where $\omega$ is a primitive fourth root of unity. The field $\mathbb{Q}(\zeta)$ contains either $\sqrt{p}$ or $\sqrt{-p}$, depending on the value of $p$ mod. 4. The existence of $\omega$ allows us to assume that $\sqrt{p} \in K$ and treat our computations in a unified way avoiding the distinction of cases.

The proof of Theorem 2 is quite involved. We will divide it into several parts. 
5.1. Elements that must be in any Hopf order. Suppose that $X$ is a Hopf order of $H$ over $R$. Our goal in this first part is to prove that several elements of $H$, arising from (co)characters, must belong to $X$. This will be used later to show that all basis elements of $H$, given in (4.1), must be in $X$.

We retain the notation of Section 3, $e_{0}, e_{1}$ are the units of $A_{0}$ and $A_{1}$ and $\varepsilon_{A}, \varepsilon_{g A}$ denote the counits of $A$ and $g A$ respectively. We start with the following:

Lemma 5.1. The elements $e_{0}, e_{1}$ are in $X$ and $\varepsilon_{A}, \varepsilon_{g A}$ are in $X^{\star}$.

Proof. We first show that $e_{0}, e_{1} \in X$. The subalgebra $H_{b}$ of $H$ generated by $u_{b}$ and $v_{b}$ is a Hopf subalgebra. Consider the algebra maps $\sigma: H_{b} \rightarrow K, u_{b} \mapsto \zeta, v_{b} \mapsto 0$ and $\tau: H_{b} \rightarrow K, u_{b} \mapsto 0, v_{b} \mapsto \zeta$. They are group-like elements of $H_{b}^{*}$ and $\sigma^{2}=\tau^{p}=1$ and $\sigma \tau=\tau^{p-1} \sigma$. Then $H_{b}^{*} \simeq K\left(C_{2} \ltimes C_{p}\right)$ as Hopf algebras and $X \cap H_{b}$ may be viewed as a Hopf order of $K\left(C_{2} \ltimes C_{p}\right)^{*}$ by Proposition 1.1(iii). According to the proof of [1, Proposition 2.1], $X \cap H_{b}$ contains the idempotents $t_{0}, t_{1}$ (notation as there). Let $\left\{\nu_{\sigma^{i} \tau^{j}}\right\}_{i, j} \subset K\left(C_{2} \ltimes C_{p}\right)^{*}$ be the dual basis of $\left\{\sigma^{i} \tau^{j}\right\}_{i, j}$. Recall that $t_{0}=\sum_{j} \nu_{\tau^{j}}$ and $t_{1}=\sum_{j} \nu_{\sigma \tau^{j}}$. One can verify directly that $\nu_{\tau^{j}}=\frac{1}{p} \sum_{k} \zeta^{-j k} u_{b}^{k}$ and $\nu_{\sigma \tau^{j}}=\frac{1}{p} \sum_{k} \zeta^{(j-1) k} v_{b}^{k}$. Then $t_{0}=e_{0}$ and $t_{1}=e_{1}$.

For the second statement, take into account that $H$ is self-dual by Proposition 4.2. The isomorphism between $H$ and $H^{*}$ established there maps $e_{0}, e_{1}$ to $\varepsilon_{A}, \varepsilon_{g A}$ respectively, see (4.11). We now get that $\varepsilon_{A}, \varepsilon_{g A} \in X^{\star}$ from self-duality of $H$, the above fact, and the first statement applied to $X^{\star}$ and $H^{*}$.

Recall from (4.7) the element $B$ used in describing $H^{*}$.

Lemma 5.2. The elements ge ${ }_{1}$ and $B$ belong to $X$ and $X^{\star}$ respectively.

Proof. We first prove that $g e_{1} \in X$. We know from Proposition 1.2 that characters of $H^{*}$ are in $X$ and characters of $H$ are in $X^{\star}$. Using the previous lemma, (4.17) and (4.14) we obtain that

$$
\begin{gathered}
\Gamma_{1}:=e_{0} \psi_{N^{+}}=\frac{1}{p} \sum_{i, j} g u_{a}^{i} u_{b}^{j} \in X, \\
\Gamma_{2}:=\varepsilon_{g A} \chi_{M^{+}}=\frac{1}{\sqrt{p}} \sum_{k} \gamma_{k k} B \in X^{\star} .
\end{gathered}
$$

Then $\left(\Gamma_{2} \otimes_{R} i d_{X}\right) \Delta\left(\Gamma_{1}\right) \in X$. We check that $\left(\Gamma_{2} \otimes_{R} i d_{X}\right) \Delta\left(\Gamma_{1}\right)=g e_{1}$. Recall that $\Gamma_{2}$ vanishes on $A_{0} \oplus A_{1} \oplus g A_{0}$, so we only need to compute the part of $\Delta\left(\Gamma_{1}\right)$ in 
$g A_{1} \otimes g A_{1}$. It is:

$$
\begin{aligned}
& \frac{1}{p^{2}} \sum_{i, j, k, l}\left(g v_{a}^{k} v_{b}^{l} \otimes g v_{a}^{-l} v_{b}^{k}\right)\left(v_{a}^{i} v_{b}^{j} \otimes v_{a}^{i} v_{b}^{-j}\right) \\
& =\frac{1}{p^{2}} \sum_{i, j, k, l} \zeta^{-i(k+l)} g v_{a}^{k+i} v_{b}^{l+j} \otimes g v_{a}^{i-l} v_{b}^{k-j} \\
& =\frac{1}{p^{2}} \sum_{i^{\prime}, j^{\prime}, k, l^{\prime}} \zeta^{\left(l^{\prime}-i^{\prime}\right)\left(i^{\prime}-k\right)} g v_{a}^{i^{\prime}} v_{b}^{j^{\prime}} \otimes g v_{a}^{l^{\prime}} v_{b}^{i^{\prime}-j^{\prime}-l^{\prime}} \\
& =\frac{1}{p} \sum_{i, j, l} \zeta^{(l-i) i}\left(\frac{1}{p} \sum_{k} \zeta^{(i-l) k}\right) g v_{a}^{i} v_{b}^{j} \otimes g v_{a}^{l} v_{b}^{i-j-l} \\
& =\frac{1}{p} \sum_{i, j} g v_{a}^{i} v_{b}^{j} \otimes g v_{a}^{i} v_{b}^{-j} . \quad \operatorname{putting} i=i^{\prime}, j=j^{\prime}, \text { and } l=l^{\prime},
\end{aligned}
$$

Applying $\Gamma_{2} \otimes_{R} i d_{X}$ to this expression we get

$$
\begin{aligned}
\frac{1}{p \sqrt{p}} \sum_{i, j, k}\left(\gamma_{k k} B\right)\left(g v_{a}^{i} v_{b}^{j}\right) g v_{a}^{i} v_{b}^{-j} \stackrel{\text { (4.8) }}{=} \frac{1}{p} \sum_{i, k} \zeta^{i k} g v_{a}^{i} \\
=\sum_{i}\left(\frac{1}{p} \sum_{k} \zeta^{i k}\right) g v_{a}^{i} \\
=g e_{1} .
\end{aligned}
$$

Therefore $g e_{1} \in X$.

We next show that $B \in X^{\star}$. From (4.14) and Proposition 1.2, we know that $\chi_{M^{+}}=p t_{00}+\frac{1}{\sqrt{p}} \sum_{i} \gamma_{i i} B \in X^{\star}$. Using Lemma 5.1, we obtain $\varepsilon_{A} \chi_{M^{+}}=p t_{00} \in X^{\star}$. Now,

$$
\left(\varepsilon_{g A} \otimes \varepsilon_{g A}\right) \Delta\left(p t_{00}\right) \stackrel{(4.6)}{=} \frac{1}{p} \sum_{k, l} \gamma_{k l} B \otimes \gamma_{l k} B \in X^{\star} \otimes_{R} X^{\star} .
$$

On the other hand, by (4.17) and Proposition 1.2, we have

$$
\psi_{N^{+}}=\frac{1}{p} \sum_{i, j} g u_{a}^{i} u_{b}^{j}+\frac{1}{\sqrt{p}} \sum_{i} g v_{a}^{i} \in X .
$$

Using again Lemma 5.1, we get

$$
e_{1} \psi_{N^{+}}=\frac{1}{\sqrt{p}} \sum_{i} g v_{a}^{i} \in X .
$$

Finally, applying $e_{1} \psi_{N^{+}} \otimes_{R} i d_{X^{\star}}$ to (5.2) we obtain

$$
\frac{1}{p \sqrt{p}} \sum_{i, k, l}\left(\gamma_{k l} B\right)\left(g v_{a}^{i}\right) \gamma_{l k} B=\frac{1}{p} \sum_{i, k, l} \zeta^{i l} \delta_{l-k, 0} \gamma_{l k} B=\gamma_{00} B=B .
$$

So, $B \in X^{\star}$.

Lemma 5.3. The elements $u_{a}, v_{a}, \frac{1}{\sqrt{p}} \sum_{i} u_{a}^{i}$, and $\frac{1}{\sqrt{p}} \sum_{i} v_{a}^{i}$ belong to $X$. 
Proof. By (4.15) and Proposition 1.2, $u_{a}+v_{a} \in X$. Then $e_{1}\left(u_{a}+v_{a}\right)=v_{a} \in X$ and $u_{a}=\left(u_{a}+v_{a}\right)-v_{a} \in X$. We have just seen in the above proof that $\frac{1}{\sqrt{p}} \sum_{i} g v_{a}^{i} \in X$. Multiplying by $g e_{1}$, we have $\frac{1}{\sqrt{p}} \sum_{i} v_{a}^{i} \in X$. Let $H_{a}$ be the Hopf subalgebra of $H$ generated by $u_{a}$ and $v_{a}$. Proposition 1.1(iii) entails that $X \cap H_{a}$ is a Hopf order of $H_{a}$. Then

$$
\Delta\left(\frac{1}{\sqrt{p}} \sum_{i} v_{a}^{i}\right) \stackrel{(3.1)}{=} \frac{1}{\sqrt{p}} \sum_{i} u_{a}^{i} \otimes v_{a}^{i}+v_{a}^{i} \otimes u_{a}^{i} \in\left(X \cap H_{a}\right) \otimes_{R}\left(X \cap H_{a}\right) .
$$

Consider the character $\varphi$ of $H_{a}$ given by $\varphi\left(u_{a}\right)=0$ and $\varphi\left(v_{a}\right)=1$. By Proposition 1.2. $\varphi \in\left(X \cap H_{a}\right)^{\star}$. Applying $\varphi \otimes_{R} i d_{X \cap H_{a}}$ to the above equality we conclude that $\frac{1}{\sqrt{p}} \sum_{i} u_{a}^{i} \in X$.

5.2. A special case. If we show that $g e_{0} \in X$, then it will follow from Lemmas 5.1 , 5.2, and 5.3, that all elements of the basis $\mathcal{B}$ in (4.1) of $H$ will be in any Hopf order $X$. Unlike for other elements, this can not be shown directly. The strategy will be to adjoin to $K$ an element $\pi$ such that $\pi^{2}=\zeta-1$, prove the statement in this case and then derive it for $K$. So, in this subsection we assume that $K$ contains such an element $\pi$. The proof requires some preparations.

Lemma 5.4. The map $T: A_{1} \rightarrow g A_{0}, v_{a}^{i} v_{b}^{j} \mapsto\left(B \otimes_{R} i d_{X}\right) \Delta\left(g v_{a}^{i} v_{b}^{j}\right)$ can be expressed as

$$
T\left(v_{a}^{i} v_{b}^{j}\right)=\frac{1}{\sqrt{p}} \sum_{k} \zeta^{j k} g u_{a}^{k} u_{b}^{i-k}
$$

Moreover, $T\left(X \cap A_{1}\right) \subseteq X \cap\left(g A_{0}\right)$.

Proof. Since $B$ vanishes on $A_{0} \oplus A_{1} \oplus g A_{0}$, only the part of $\Delta\left(g v_{a}^{i} v_{b}^{j}\right)$ in $g A_{1} \otimes g A_{0}$ is relevant for the computation. We have:

$$
\begin{aligned}
T\left(v_{a}^{i} v_{b}^{j}\right) & \stackrel{\text { Th. }}{=} \frac{1}{p} \sum_{k, l} \zeta^{k(k+l)} B\left(g v_{a}^{k+l} v_{b}^{-(k+l)} v_{a}^{i} v_{b}^{j}\right) g u_{a}^{k+i} u_{b}^{l-j} \\
& =\frac{1}{p} \sum_{k, l} \zeta^{(k+i)(k+l)} B\left(g v_{a}^{k+l+i} v_{b}^{-(k+l)+j}\right) g u_{a}^{k+i} u_{b}^{l-j} \\
& \stackrel{(4.8)}{=} \frac{1}{\sqrt{p}} \sum_{k} \zeta^{(k+i) j} g u_{a}^{k+i} u_{b}^{-k} \\
& =\frac{1}{\sqrt{p}} \sum_{k} \zeta^{k j} g u_{a}^{k} u_{b}^{i-k} .
\end{aligned}
$$

Let now $x \in X \cap A_{1}$. By Lemma [5.2, we know that $g e_{1} \in X$ and $B \in X^{\star}$. Then $g x=g e_{1} x \in X$ and $\Delta(g x) \in X \otimes_{R} X$. From here, $T(x)=\left(B \otimes_{R} i d_{X}\right) \Delta(g x) \in X$.

Proposition 5.5. Let $Z$ be an R-algebra and $z, e \in Z$. Assume that $z e=e z=z$. Set $\tilde{z}=\frac{1}{\pi}(z-e)$. If $\tilde{z} \in Z$, then

$$
\frac{1}{\sqrt{p}} \sum_{i} z^{i}
$$

is an R-linear combination of powers of $\tilde{z}$. 
Proof. Set

$$
\frac{(\pi \tilde{z}+e)^{p}-e}{\pi \tilde{z}}=\sum_{k=1}^{p}\left(\begin{array}{l}
p \\
k
\end{array}\right)(\pi \tilde{z})^{k-1}
$$

As in the proof of Lemma 2.3, the fractional expression is just symbolic. The lefthand side equals $\sum_{i=0}^{p-1} z^{i}$. We obtain the result by dividing this equation by $\sqrt{p}$, noticing that $\pi^{p-1}=\xi \sqrt{p}$ for some invertible $\xi \in R$, and $\left(\begin{array}{l}p \\ k\end{array}\right)$ is divisible by $p$ for any $k=1, \ldots, p-1$.

We are now ready to tackle the difficult point.

Lemma 5.6. The element ge $e_{0}$ belongs to $X$.

Proof. View $A$ as a Hopf subalgebra of $H$ and $A_{0}$ as a quotient Hopf algebra of $A$ via projecting any element on its component in $A_{0}$. Then $X \cap A_{0}$ is a Hopf order of $A_{0}$ in light of Proposition 1.1. Look now at the Hopf subalgebra of $A_{0}$ generated by $u_{a}$. Lemma 5.3 shows that $\frac{1}{\sqrt{p}} \sum_{i} u_{a}^{i} \in X$. Applying Corollary 2.9(ii), we have $\frac{1}{\pi}\left(u_{a}-e_{0}\right) \in X \cap A_{0}$.

On the other hand, Lemmas 5.1 and 5.4 yield that

$$
T\left(e_{1}\right)=\frac{1}{\sqrt{p}} \sum_{k} g u_{a}^{k} u_{b}^{-k} \in X .
$$

Put $e=\frac{1}{p} \sum_{k} u_{a}^{k} u_{b}^{-k}$. Observe that $e$ is an idempotent and $T\left(e_{1}\right)=\sqrt{p} g e \in X$. Let $G$ be the group generated by $\sigma, \tau$ subject to $\sigma^{2}=\tau^{p}=1, \sigma \tau=\tau \sigma$. The assignments $e_{1} \mapsto 0 ; u_{a}, u_{b} \mapsto \tau ; g \mapsto \sigma$ define a surjective algebra map $f: H \rightarrow K G$. It is easy to check that $f$ is a Hopf algebra map and Ker $f$ equals the ideal generated by $e_{1}$ and $u_{a} u_{b}^{-1}-e_{0}$. By Proposition 1.1(iv), $f(X)$ is a Hopf order of $K G$. The element $\sigma$ must be in $f(X)$ because it can be received from characters of $(K G)^{*}$. Take $x \in X \cap A_{0}$ such that $f(x)=\sigma$. Then $x-g e_{0}=h\left(u_{a} u_{b}^{-1}-e_{0}\right)$ for some $h \in H$. Multiplying by $\sqrt{p} g e$ we arrive to $\sqrt{p}(x g e-e)=0$. Thus $\sqrt{p} e=x(\sqrt{p} g e) \in X \cap A_{0}$. Consider the Hopf subalgebra $E$ of $A_{0}$ generated by $u_{a} u_{b}^{-1}$. As $\sqrt{p} e=\frac{1}{\sqrt{p}} \sum_{k} u_{a}^{k} u_{b}^{-k} \in X \cap E$, Corollary 2.9(i) implies $\frac{1}{\pi}\left(u_{a} u_{b}^{-1}-e_{0}\right) \in X$. Hence

$$
\frac{1}{\pi}\left(u_{b}^{-1}-e_{0}\right)=u_{a}^{-1}\left(\frac{1}{\pi}\left(u_{a} u_{b}^{-1}-e_{0}\right)-\frac{1}{\pi}\left(u_{a}-e_{0}\right)\right) \in X .
$$

By Proposition 5.5, $\frac{1}{\sqrt{p}} \sum_{i} u_{b}^{i} \in X$. Let $H_{b}$ be the Hopf subalgebra of $H$ generated by $u_{b}$ and $v_{b}$. Arguing as we did for $H_{a}$ in the proof of Lemma 5.3, we obtain that $\frac{1}{\sqrt{p}} \sum_{i} v_{b}^{i} \in X$. Applying Lemma [5.4, we have

$$
T\left(\frac{1}{\sqrt{p}} \sum_{i} v_{b}^{i}\right)=g e_{0} \in X
$$

and we are done.

5.3. The necessary condition. We next derive that all basis elements of $H$ must be in the Hopf order $X$. This will be key to establish the necessary condition of our main result and to prove later that a Hopf order of $H$, if exists, must be unique.

Proposition 5.7. All elements of the basis $\mathcal{B}$ in (4.1) of $H$ belong to $X$. 
Proof. From Lemmas 5.1 and 5.3, we know that $e_{0}, e_{1}, u_{a}, v_{a} \in X$. We next see that $g \in X$. Take $\pi \in \mathbb{C}$ such that $\pi^{2}=\zeta-1$ and set $L=K(\pi), S=\mathcal{O}_{L}$. Then $X \otimes_{R} S$ is a Hopf order of $H_{L}:=H \otimes_{K} L$. Lemma 5.2 combined with Lemma 5.6 yields that $g \in X \otimes_{R} S$. We can identify $H \otimes_{R} L$ with $H_{L}$ via multiplication. Inside $H \otimes_{R} L$ we have $(X+R g) \otimes_{R} S \subseteq X \otimes_{R} S+R g \otimes_{R} S=X \otimes_{R} S \subseteq(X+R g) \otimes_{R} S$. This equality holds indeed in $H \otimes_{R} S \subset H \otimes_{R} L$. Since $S$ is faithfully flat as an $R$-module, we obtain $X=X+R g$. Therefore $g \in X$.

It remains to prove that $u_{b}, v_{b} \in X$. We have that $u_{b} g=g u_{a} \in X$. Then $u_{b}=\left(u_{b} g\right) g \in X$ and consequently $\Delta\left(u_{b}\right) \in X \otimes_{R} X$. If follows from the latter that $v_{b} \in X$ arguing for $H_{b}$ as we did for $H_{a}$ in the proof of Lemma 5.3.

As a consequence of Lemma 5.3, we get

$$
\frac{1}{\sqrt{p}} \sum_{i} u_{a}^{i}+v_{a}^{i} \in X
$$

Let $E$ be the Hopf subalgebra of $H$ generated by the group-like element $h:=u_{a}+v_{a}$. Clearly, $E \simeq K C_{p}$ as Hopf algebras. Put $Z=E \cap X$ and denote by $\Lambda$ the set of left integrals in the Hopf order $Z$ of $E$.

Lemma 5.8. We have $\Lambda=R\left(\frac{1}{\sqrt{p}} \sum_{i} h^{i}\right)$.

Proof. Obviously, $R\left(\frac{1}{\sqrt{p}} \sum_{i} h^{i}\right) \subseteq \Lambda$. For the reverse inclusion, let $\int \in \Lambda$. There is $\lambda \in K$ such that $\int=\frac{\lambda}{\sqrt{p}} \sum_{i} h^{i}$. We will prove that $\lambda \in R$. Using Proposition 5.7 . $\varpi:=\left(\int \otimes \int\right) \Delta(g) \in X \otimes_{R} X$. Then $\left(\Gamma_{2} \otimes_{R} \Gamma_{2}\right)(\varpi) \in R$, with $\Gamma_{2}$ being the element defined in (5.1). We next show that $\left(\Gamma_{2} \otimes_{R} \Gamma_{2}\right)(\varpi)=\lambda^{2}$.

Taking into account that $\Gamma_{2}$ vanishes on $A_{0} \oplus A_{1} \oplus g A_{0}$, it suffices to compute the part of $\varpi$ in $g A_{1} \otimes g A_{1}$. We have:

$$
\begin{aligned}
\left(\Gamma_{2} \otimes_{R} \Gamma_{2}\right)(\varpi) & \stackrel{\text { 5.1) }}{=} \frac{\lambda^{2}}{p^{3}} \sum_{i, j, k, l} \sum_{r, s}\left(\gamma_{r r} B\right)\left(g v_{a}^{i+k} v_{b}^{l}\right)\left(\gamma_{s s} B\right)\left(g v_{a}^{j-l} v_{b}^{k}\right) \\
& \stackrel{(4.8)}{=} \frac{\lambda^{2}}{p^{2}} \sum_{i, j, k, l} \sum_{r, s} \zeta^{r(i+k)} \delta_{l, 0} \zeta^{s(j-l)} \delta_{k, 0} \\
& =\lambda^{2} .
\end{aligned}
$$

So $\lambda^{2} \in R$ and thus $\lambda \in R$.

We can now establish the necessary condition in our main result from the previous lemma and Corollary 2.9(i):

Proposition 5.9. Suppose that $H$ admits a Hopf order over $R$. Then there is an ideal $I$ of $R$ such that $I^{2(p-1)}=(p)$.

5.4. The Hopf order. Assume that there is an ideal $I$ of $R$ such that $I^{2(p-1)}=(p)$. In this subsection we will construct from $I$ a Hopf order of $H$ which will turn out to be the only Hopf order. Consider the fractional ideal $J:=I^{-1}=\{\alpha \in K: \alpha I \subseteq R\}$. By the unique factorization property in $R$, from $I^{2(p-1)}=(p)=(\zeta-1)^{p-1}=(\sqrt{p})^{2}$, it follows that $I^{2}=(\zeta-1)$ and $I^{p-1}=(\sqrt{p})$. Then $J^{2}=\left(\frac{1}{\zeta-1}\right)$ and $J^{p-1}=\left(\frac{1}{\sqrt{p}}\right)$.

We need the following version of Proposition 5.5 
Proposition 5.10. Let $Z$ be an R-algebra and $z, e \in Z$. Assume that $z e=e z=z$. If $J(z-e) \subset Z$, then

$$
\frac{1}{\sqrt{p}} \sum_{i} z^{i} \in Z
$$

Proof. Put $\tilde{z}=z-e$, proceed like in the other proof and use that $\frac{1}{\sqrt{p}} \tilde{z}^{p-1} \in Z$.

Theorem 5.11. The $R$-subalgebra $Y$ of $H$ generated by $e_{0}, e_{1}, g, J\left(u_{a}-e_{0}\right)$, $J\left(u_{b}-e_{0}\right), J\left(v_{a}-e_{1}\right)$, and $J\left(v_{b}-e_{1}\right)$ is a Hopf order of $H$.

Proof. We will first prove that $Y$ is finitely generated as an $R$-module. Observe that $J$ is finitely generated. Write

$$
x_{a}=u_{a}-e_{0}, \quad x_{b}=u_{b}-e_{0}, \quad y_{a}=v_{a}-e_{1}, \quad y_{b}=v_{b}-e_{1} .
$$

We have that $x_{a}, x_{b}, y_{a}, y_{b} \in Y$ because $I J=R$. Since $e_{0}, e_{1} \in Y$, we also have $u_{a}, u_{b}, v_{a}, v_{b} \in Y$. We next check that $\left(J x_{a}\right)^{n} \subset \sum_{i=1}^{p-1} J^{i} x_{a}^{i}$ for $n \geq p$. The element $x_{a}$ satisfy $\sum_{i=1}^{p}\left(\begin{array}{c}p \\ i\end{array}\right) x_{a}^{i}=0$. As $J^{p} J^{p-2}=J^{2(p-1)}=\left(\frac{1}{p}\right)$, we get $R=\left(J^{p} p\right) J^{p-2}$. Then $J^{p} p=I^{p-2} \subset R$. Hence

$$
\left(J x_{a}\right)^{p}=J^{p} x_{a}^{p} \subset \sum_{i=1}^{p-1} J^{p}\left(\begin{array}{c}
p \\
i
\end{array}\right) x_{a}^{i} \subset \sum_{i=1}^{p-1} R x_{a}^{i} \subset \sum_{i=1}^{p-1} J^{i} x_{a}^{i} .
$$

The same holds for $x_{b}, y_{a}$, and $y_{b}$. Consider now the equality:

$$
\begin{aligned}
y_{a} y_{b} & =v_{a} v_{b}-v_{a}-v_{b}+e_{1} \\
& =\zeta v_{b} v_{a}-v_{a}-v_{b}+e_{1} \\
& =\zeta y_{b} y_{a}+(\zeta-1)\left(y_{a}+y_{b}+e_{1}\right) .
\end{aligned}
$$

Then, for $\alpha_{a}, \alpha_{b} \in J$ the coefficient of $e_{1}$ in $\left(\alpha_{a} y_{a}\right)\left(\alpha_{b} y_{b}\right)$ belongs to $R$ because $J^{2}=\left(\frac{1}{\zeta-1}\right)$. Using the previous equality one can prove that any product of the form $\left(\beta_{a} y_{a}^{k}\right)\left(\beta_{b} y_{b}^{l}\right)$ with $\beta_{a} \in J^{k}, \beta_{b} \in J^{l}$ can be expressed as an $R$-linear combination of elements in $\left(J^{i} y_{b}^{i}\right)\left(J^{j} y_{a}^{j}\right)$ with $0 \leq i \leq l, 0 \leq j \leq k$. Notice that the coefficient of $e_{1}$ always belong to $R$. All these facts, together with the relations among $x_{a}, x_{b}, y_{a}, y_{b}$, and $g$ inside $H$, show that $Y$ is finitely generated as an $R$-module. More precisely, using that $J$ is finitely generated, the following elements generate $Y$ over $R$ :

$e_{0}, e_{1}, g e_{0}, g e_{1}, J^{i+j}\left(x_{b}^{i} x_{a}^{j}\right), J^{i+j}\left(g x_{b}^{i} x_{a}^{j}\right), J^{i+j}\left(y_{b}^{i} y_{a}^{j}\right), J^{i+j}\left(g y_{b}^{i} y_{a}^{j}\right)$,

$$
i, j=0, \ldots, p-1 \text {. }
$$

Removing the powers of $J$ from these elements, we obtain a $K$-basis of $H$ (we understand that $i, j$ are not simultaneously zero). Hence $Y$ is an order of $H$.

We next prove that $Y$ is closed under comultiplication and antipode. It is easy to check that the comultiplication of the $e$ 's, $x$ 's and $y$ 's lie in $Y \otimes_{R} Y$, the counits of them lies in $R$, and $S(Y) \subset Y$. For instance, for $\alpha \in J$ we have:

$$
\begin{aligned}
\Delta\left(\alpha x_{a}\right)= & \alpha x_{a} \otimes u_{a}+\alpha y_{a} \otimes v_{a}+e_{0} \otimes \alpha x_{a}+e_{1} \otimes \alpha y_{a} \in Y \otimes_{R} Y, \\
\Delta\left(\alpha x_{b}\right)= & \alpha x_{b} \otimes u_{b}+e_{0} \otimes \alpha x_{b}+\alpha y_{b} \otimes v_{b}^{p-1} \\
& +\sum_{k=1}^{p-1} e_{1} \otimes\left(\begin{array}{c}
p-1 \\
k
\end{array}\right)\left(\alpha y_{b}\right) y_{b}^{k-1} \in Y \otimes_{R} Y .
\end{aligned}
$$


It only remains to show that $\Delta(g) \in Y \otimes_{R} Y$. For, we need to rewrite $\Delta(g)$ as an $R$-linear combination of elements in $Y \otimes_{R} Y$. Recall from Equation 3.2 that $\Delta(g)$ consists of four summands. We treat each of them separately:

- Part in $A_{0} \otimes A_{0}$. Consider the sum

$$
\frac{1}{p^{2}} \sum_{i, j, k, l} \zeta^{j k-i l} u_{a}^{i} u_{b}^{j} \otimes u_{a}^{k} u_{b}^{l}=\left(\frac{1}{p} \sum_{i, l} \zeta^{-i l} u_{a}^{i} \otimes u_{b}^{l}\right)\left(\frac{1}{p} \sum_{j, k} \zeta^{j k} u_{b}^{j} \otimes u_{a}^{k}\right) .
$$

We argue on the first factor, the second one being similar. Replace $u_{a}$ and $u_{b}$ by $x_{a}+e_{0}$ and $x_{b}+e_{0}$ respectively and expand. The coefficient of $x_{a}^{r} \otimes x_{b}^{s}$ equals $\frac{\zeta^{-1}}{p}$ if $r=s=p-1$. Then

$$
\frac{\zeta^{-1}}{p} x_{a}^{p-1} \otimes x_{b}^{p-1}=\frac{\zeta^{-1}}{\sqrt{p}} x_{a}^{p-1} \otimes \frac{1}{\sqrt{p}} x_{b}^{p-1}
$$

belongs to $Y \otimes_{R} Y$ because $\frac{1}{\sqrt{p}} \in J^{p-1}$. For either $r$ or $s$ different from $p-1$ we use the following argument. The coefficient of $x_{a}^{r} \otimes x_{b}^{s}$ will be the same as the coefficient of $y_{a}^{r} \otimes y_{b}^{s}$ in the sum

$$
\frac{1}{p} \sum_{i, l} \zeta^{-i l} v_{a}^{i} \otimes v_{b}^{l}
$$

This in turn will be the same as the coefficient of $y_{a}^{r} y_{b}^{s}$ in the sum

$$
\begin{aligned}
\frac{1}{p} \sum_{i, l} \zeta^{-i l} v_{a}^{i} v_{b}^{l} & =\frac{1}{p} \sum_{i, l} v_{b}^{l} v_{a}^{i} \\
& =\frac{1}{p}\left(\sum_{l} v_{b}^{l}\right)\left(\sum_{i} v_{a}^{i}\right) \\
& =\frac{1}{p} \frac{\left(y_{b}+e_{1}\right)^{p}-e_{1}}{y_{b}} \frac{\left(y_{a}+e_{1}\right)^{p}-e_{1}}{y_{a}} .
\end{aligned}
$$

We are using here the convention in the proof of Proposition 2.3 for these fractional expressions. The coefficient of $y_{a}^{r} y_{b}^{s}$ in this sum contains the binomial coefficient $\left(\begin{array}{l}p \\ k\end{array}\right)$ for $k=1, \ldots, p-1$. Therefore the first factor belong to $Y \otimes_{R} Y$.

- $\underline{\text { Part in } A_{0} \otimes A_{1}}$. We have the summand

$$
\frac{1}{p} \sum_{k, l} \zeta^{-(k+l) k} u_{a}^{k} u_{b}^{l} \otimes v_{a}^{k+l} v_{b}^{k+l}=\left(\frac{1}{\sqrt{p}} \sum_{k} u_{a}^{k} \otimes v_{b}^{k} v_{a}^{k}\right)\left(\frac{1}{\sqrt{p}} \sum_{l} u_{b}^{l} \otimes v_{a}^{l} v_{b}^{l}\right) .
$$

We show that each of the sums belongs to $Y \otimes_{R} Y$. We do it only for the first one. For the second one proceed similarly. The coefficient of $y_{a}^{r} \otimes y_{b}^{s} y_{a}^{t}$ in this sum will be the same as the coefficient of $x_{a}^{r} \otimes x_{b}^{s} x_{a}^{t}$ in the sum

$$
\frac{1}{\sqrt{p}} \sum_{k} u_{a}^{k} \otimes u_{b}^{k} u_{a}^{k}=\frac{1}{\sqrt{p}} \sum_{k}\left(u_{a} \otimes u_{b} u_{a}\right)^{k} .
$$

Observe that $u_{a} \otimes u_{b} u_{a} \in Y \otimes_{R} Y$ and

$$
\begin{aligned}
J\left(u_{a} \otimes u_{b} u_{a}-e_{0} \otimes e_{0}\right)= & J x_{a} \otimes x_{b} x_{a}+J x_{a} \otimes x_{b}+J x_{a} \otimes x_{a}+J x_{a} \otimes e_{0} \\
& +e_{0} \otimes\left(J x_{b}\right) x_{a}+e_{0} \otimes J x_{b}+e_{0} \otimes J x_{a} \in Y \otimes_{R} Y .
\end{aligned}
$$

This together Proposition 5.10 yields that the sum belongs to $Y \otimes_{R} Y$. 
- Part in $A_{1} \otimes A_{0}$. We argue as before with the summand

$\frac{1}{p} \sum_{k, l} \zeta^{(k+l) k} v_{a}^{k+l} v_{b}^{-(k+l)} \otimes u_{a}^{k} u_{b}^{l}=\left(\frac{1}{\sqrt{p}} \sum_{k} v_{b}^{(p-1) k} v_{a}^{k} \otimes u_{a}^{k}\right)\left(\frac{1}{\sqrt{p}} \sum_{l} v_{a}^{l} v_{b}^{(p-1) l} \otimes u_{b}^{l}\right)$.

but using the following variation: $v_{b}^{p-1}=\bar{y}_{b}+e_{1}$ with $\bar{y}_{b}=\sum_{j=1}^{p-1}\left(\begin{array}{c}p-1 \\ j\end{array}\right) y_{b}^{j}$ and $J \subset J^{j}$ for $j=1, \ldots, p-1$.

- $\underline{\text { Part in } A_{1} \otimes A_{1}}$. Consider the summand

$$
\frac{1}{p} \sum_{k, l} v_{a}^{k} v_{b}^{l} \otimes v_{a}^{-l} v_{b}^{k}=\frac{1}{p} \sum_{k, l} v_{a}^{k} v_{b}^{l} \otimes v_{a}^{(p-1) l} v_{b}^{k}
$$

Write it in $H \otimes H^{o p}$ as

$$
\left(\frac{1}{\sqrt{p}} \sum_{k} v_{a}^{k} \otimes v_{b}^{k}\right)\left(\frac{1}{\sqrt{p}} \sum_{l} v_{b}^{l} \otimes v_{a}^{(p-1) l}\right)
$$

and proceed as before. This finishes the proof.

Proposition 5.12. The Hopf order $Y$ is unique.

Proof. Let $\pi \in \mathbb{C}$ be such that $\pi^{2}=\zeta-1$ and set $L=K(\pi)$. We will first prove that $H_{L}$ admits a unique Hopf order over $S=\mathcal{O}_{L}$ and derive the uniqueness for $H$ arguing as we did in Proposition 5.7. Write $I=(\pi)$. Then $I^{2(p-1)}=(p)$. Let $J \subset L$ be the inverse of $I$, which is generated by $\frac{1}{\pi}$. We have seen in the precedent proof that the order $Y$ (over $S$ ) is generated as an algebra by $e_{0}, e_{1}, g$ and the elements

$$
\tilde{x}_{a}:=\frac{1}{\pi}\left(u_{a}-e_{0}\right), \quad \tilde{x}_{b}:=\frac{1}{\pi}\left(u_{b}-e_{0}\right), \quad \tilde{y}_{a}:=\frac{1}{\pi}\left(v_{a}-e_{1}\right), \quad \tilde{y}_{b}:=\frac{1}{\pi}\left(v_{b}-e_{1}\right) .
$$

Let $X$ be any Hopf order of $H_{L}$. By Lemma 5.3 and Corollary [2.9)(ii), $X$ must contain the element $\frac{1}{\pi}\left(u_{a}+v_{a}-1\right)$. By Proposition 5.7 $X$ contains all basis elements of $H_{L}$. Using multiplication by $e_{0}$ and $e_{1}$, conjugation by $g$ and translation by the character $\rho: H_{b} \rightarrow K, u_{b} \mapsto 0, v_{b} \mapsto 1$, we see that $X$ must contain $\tilde{x}_{a}, \tilde{y}_{a}, \tilde{x}_{b}$, and $\tilde{y}_{b}$. Then $Y \subseteq X$ and thus $Y$ is a minimal Hopf order.

We know that $H_{L}$ is self-dual. Then $H_{L}^{*}$ has also a minimal order, which we denote by $Z$. This implies that $Z^{\star}$ is a maximal Hopf order of $H_{L}$. Thus any Hopf order of $H_{L}$ lies between $Y$ and $Z^{\star}$. We will prove that $Y=Z^{\star}$. The $R$-submodule $\Lambda_{Y}$ of left integrals in $Y$ is spanned by $\frac{1}{p}(1+g) \sum_{i, j} u_{a}^{i} u_{b}^{j}$. Then $\varepsilon\left(\Lambda_{Y}\right)=(2 p)$. Using self-duality of $H_{L}$, we also have $\varepsilon\left(\Lambda_{Z}\right)=(2 p)$. Since $(\operatorname{dim} H)=\left(4 p^{2}\right)$, by Proposition 1.3. $\varepsilon\left(\Lambda_{Z^{\star}}\right)=(2 p)$. Proposition 1.4 yields $Y=Z^{\star}$.

Finally, let $X, X^{\prime}$ be two Hopf orders of $H$. The Hopf orders $X \otimes_{R} S$ and $X^{\prime} \otimes_{R} S$ of $H_{L}$ must be equal. Then $X \otimes_{R} S=\left(X+X^{\prime}\right) \otimes_{R} S=X^{\prime} \otimes_{R} S$. As $S$ is faithfully flat as an $R$-module, we obtain $X=X+X^{\prime}=X^{\prime}$ and we are done.

Remark 5.13. The precedent result shows that the behavior of orders for semisimple Hopf algebras can be quite different to that of group algebras. When we take larger number fields, the number of Hopf orders of the group algebra on $C_{p}$ tends to infinity whereas the number of orders of $H$ is constantly 1 . 
Remark 5.14. In [8, Theorem 1.8] the second author proved that every semisimple Hopf algebra over a number field only admits finitely many Hopf orders over its ring of integers.

5.5. Main result. We are finally in a position to prove our main result:

Theorem 5.15. Let $p$ be an odd prime number and $K$ a number field containing a primitive p-th root of unity. Nikshych's Hopf algebra admits a Hopf order over $\mathcal{O}_{K}$, which must be unique, if and only if there is an ideal $I$ of $\mathcal{O}_{K}$ such that $I^{2(p-1)}=(p)$. In particular, $K$ can not be neither a cyclotomic field nor an abelian extension of $\mathbb{Q}$ if a Hopf orders exist.

Proof. The necessary condition was established in Proposition [5.9. The sufficient condition and uniqueness were proved in Theorem 5.11 and Proposition 5.12 .

We prove that $K$ can not be a cyclotomic field if $H$ admits a Hopf order over $\mathcal{O}_{K}$. Let $I \subset \mathcal{O}_{K}$ be the given ideal such that $I^{2(p-1)}=(p)$. Suppose that $K$ is a cyclotomic field, say $K=\mathbb{Q}(\eta)$ with $\eta$ a primitive $m$-th root of unity. Since $(p)$ ramifies in $\mathcal{O}_{K}$, by [16, Proposition 2.3], $p$ is a prime factor of $m$. Call $n$ the exponent with which $p$ occurs. By [10, Theorem 4.40], there is a prime ideal $\mathfrak{P}$ of $\mathcal{O}_{K}$ appearing in the factorization of $(p)$ with exponent $e:=(p-1) p^{n-1}$. The exponent of $\mathfrak{P}$ in the factorization of $I^{2(p-1)}$ will be $2 l(p-1)$ for some $l \in \mathbb{N}$. Then $p$ should be divisible by 2 , a contradiction.

That $K$ can not be an abelian extension of $\mathbb{Q}$ in this case follows from the Kronecker-Weber Theorem.

\section{ON ORDERS OF FORMS}

Let $L / K$ be a Galois extension of fields with Galois group $\Gamma$. We have seen before that it could happen that Nikshych's Hopf algebra $H$ over $K$ does not admit an order over any cyclotomic ring of integers, but could a $L / K$-form of $H$ do? Namely, could there be another Hopf algebra $H^{\prime}$ over $K$ such that $H^{\prime} \otimes_{K} L \simeq H \otimes_{K} L$ and $H^{\prime}$ admits an order over some cyclotomic ring of integers? We will show in this last section that the answer to this question is affirmative.

We first recall from [2, Proposition 1.1] and [13, Proposition 1] some basics about Galois descent in the Hopf algebra setting. Put $H_{L}=H \otimes_{K} L$. Given $\gamma \in \Gamma$, a Hopf $\gamma$-automorphism of $H_{L}$ is a $K$-linear automorphism $f: H_{L} \rightarrow H_{L}$ which satisfies:

(1) $f$ is $\gamma$-semilinear, i.e., $f(\alpha h)=\gamma(\alpha) f(h)$ for all $\alpha \in L, h \in H_{L}$.

(2) $f$ is compatible with the multiplication, comultiplication, and antipode.

(3) $f\left(1_{H_{L}}\right)=1_{H_{L}}$.

(4) $\varepsilon f=\gamma \varepsilon$.

According to Galois descent, $L / K$-forms of $H$ correspond to group homomorphisms $\Phi: \Gamma \rightarrow \operatorname{Aut}_{K}\left(H_{L}\right), \gamma \mapsto \Phi_{\gamma}$ such that $\Phi_{\gamma}$ is a Hopf $\gamma$-automorphism for all $\gamma \in \Gamma$. For such a $\Phi$ the set of invariants $\left(H_{L}\right)^{\Gamma}$ is a Hopf algebra over $K$ and the natural map $\left(H_{L}\right)^{\Gamma} \otimes_{K} L \rightarrow H_{L}$ is an isomorphism of Hopf algebras.

Our goal is to prove the following:

Theorem 6.1. Let $\zeta_{n} \in \mathbb{C}$ be a primitive $n$-th root of unity, with $n$ divisible by $p$. Consider Nikshych's Hopf algebra $H$ as defined over $\mathbb{Q}\left(\zeta_{n}\right)$. Let $w \in \mathbb{Z}\left[\zeta_{n}\right]$ and $t \in \mathbb{C}$ 
be such that $w$ is invertible and $t^{2}=w\left(\zeta_{p}-1\right)$. Assume that there is $d \in \mathbb{Z}\left[\zeta_{n}\right]$ such

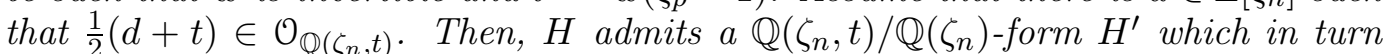
admits an order over $\mathbb{Z}\left[\zeta_{n}\right]$.

Proof. Set $L=\mathbb{Q}\left(\zeta_{n}, t\right)$. We will construct $H^{\prime}$ and show that the unique order $Y$ of $H_{L}$ descents to an order of $H^{\prime}$ over $\mathbb{Z}\left[\zeta_{n}\right]$. The Galois group $\Gamma$ of $L / \mathbb{Q}\left(\zeta_{n}\right)$ is isomorphic to $\mathbb{Z} / 2 \mathbb{Z}$. We denote the generator by $\gamma$. Bear in mind the Hopf automorphism $\sigma$ of $H$ of order two given by

$$
\sigma\left(u_{\theta}\right)=u_{\theta}^{-1}, \sigma\left(v_{\theta}\right)=v_{\theta}^{-1} \text { for } \theta=a, b, \text { and } \sigma(g)=g .
$$

We can define a Hopf $\gamma$-automorphism $\sigma^{\prime}$ of $H_{L}$ by $\sigma^{\prime}(h \otimes \alpha)=\sigma(h) \otimes \gamma(\alpha)$ for all $h \in H, \alpha \in L$. Let $\Phi: \Gamma \rightarrow \operatorname{Aut}_{K}\left(H_{L}\right)$ be the group morphism mapping $\gamma$ to $\sigma^{\prime}$. Consider the form $H^{\prime}$ of $H$ given by $H^{\prime}=\left(H_{L}\right)^{\Gamma}$.

We claim that the order $Y$ of $H_{L}$ descents to an order $Y^{\prime}:=Y^{\Gamma}$ of $H^{\prime}$ over $\mathbb{Z}\left[\zeta_{n}\right]$. It is enough to check that the natural map $\rho: Y^{\Gamma} \otimes_{\mathbb{Z}\left[\zeta_{n}\right]} \mathcal{O}_{L} \rightarrow Y$ is an isomorphism (this will ensure us that $Y^{\Gamma}$ is really a Hopf order). Since $\rho$ is injective, it suffices to check the surjectivity. We have seen in Proposition 5.12 that $Y$ is generated over $\mathcal{O}_{L}$ by $e_{0}, e_{1}, g$, and

$$
\tilde{x}_{a}:=\frac{1}{t}\left(u_{a}-e_{0}\right), \quad \tilde{x}_{b}:=\frac{1}{t}\left(u_{b}-e_{0}\right), \quad \tilde{y}_{a}:=\frac{1}{t}\left(v_{a}-e_{1}\right), \quad \tilde{y}_{b}:=\frac{1}{t}\left(v_{b}-e_{1}\right) .
$$

Clearly, $e_{0}, e_{1}, g \in \operatorname{Im} \rho$ as they are invariants. We will show that $\operatorname{Im} \rho$ contains the rest of the generators. Since $\operatorname{Im} \rho$ is a subring of $Y$, this will give $\operatorname{Im} \rho=Y$. Let us show that $\tilde{x}_{a} \in \operatorname{Im} \rho$. The proof for the other generators is similar. The element $q:=\frac{1}{t^{2}}\left(2 e_{0}-u_{a}-u_{a}^{-1}\right)=-\tilde{x}_{a}^{2} u_{a}^{-1}$ belongs to $Y^{\Gamma}$. Since $\gamma(t)=-t$, a direct calculation reveals that $\sigma^{\prime}\left(\tilde{x}_{a}\right)=\tilde{x}_{a}+t q$. Set $z=\tilde{x}_{a}+\frac{1}{2}(d+t) q$. One can easily check that $z \in Y^{\Gamma}$, and therefore $z \in \operatorname{Im} \rho$. Finally, $\tilde{x}_{a}=z-\frac{1}{2}(d+t) q$, and $\frac{1}{2}(d+t) q \in \operatorname{Im} \rho$, so $\tilde{x}_{a} \in \operatorname{Im} \rho$ as well, as desired.

With the previous theorem in hand, we will describe an example in which an order of a form does exist.

Example. Consider the case $p=7$ and $n=28$. Let $\zeta:=\zeta_{28}$ be a primitive 28 -th root of unity. A computation done by Dror Speiser with the computer algebra system MAGMA showed that if $w$ is the inverse of the element

$$
\begin{aligned}
& 21747826028152 \zeta^{11}-25061812676688 \zeta^{10}+5371269408312 \zeta^{9}-2754700868376 \zeta^{8} \\
+ & 21747826028152 \zeta^{7}-22307111808312 \zeta^{6}+4963799311635 \zeta^{4}+12069132874072 \zeta^{3} \\
- & 11153555904156 \zeta^{2}-12069132874072 \zeta+17343312496677
\end{aligned}
$$

and $d=1$, then the condition of the theorem holds. We take $t$ such that $t^{2}=w\left(1-\zeta^{4}\right)$. We thus have an order over $\mathbb{Z}[\zeta]$ of a form of $H_{7}$.

Then $H_{7}$, as defined over the complex numbers, admits an order over a cyclotomic ring of integers.

The following questions remain open: 
Questions. Does there exist a value of $p$ for which Nikshych's Hopf algebra $H_{p}$, as defined over the complex numbers, does not admit an order over any cyclotomic ring of integers? More generally, does there exist a complex semisimple Hopf algebra which admits an order over a number ring but not over any cyclotomic ring of integers?

Acknowledgements. The first author is supported by grant MTM2014-54439-P from MICINN and FEDER and by the research group FQM0211 from Junta de Andalucía. The second author was supported by the Danish National Research Foundation (DNRF) through the Centre for Symmetry and Deformation. The authors are grateful to Dror Speiser for doing the previous computer calculation and to Bjorn Poonen for a conversation about the number theoretical condition in Theorem 6.1. The authors are finally indebted to the referee for his/her comments and suggestions, which helped to improve substantially the presentation of the results.

\section{REFERENCES}

[1] J. Cuadra and E. Meir, On the existence of orders in semisimple Hopf algebras. Trans. Amer. Math. Soc. 368 (2016), 2547-2562.

[2] S. Caenepeel, S. Dascalescu, and L. Le Bruyn, Forms of pointed Hopf algebras. Manuscripta Math. 100 (1999), 35-53.

[3] C. W. Curtis and I. Reiner, Methods of representation theory. With applications to finite groups and orders. Vol. I. Pure and Applied Mathematics. John Wiley \& Sons, Inc., New York, 1981.

[4] C. Greither, Extensions of finite group schemes, and Hopf Galois theory over a complete discrete valuation ring. Math. Z. 210 (1992), 37-67.

[5] R. G. Larson, Hopf algebra orders determined by group valuations. J. Algebra 38 (1976), 414-452.

[6] R. G. Larson, Orders in Hopf algebras. J. Algebra 22 (1972), 201-210.

[7] A. Masuoka, Some further classification results on semisimple Hopf algebras. Comm. Algebra 24 (1996), 307-329.

[8] E. Meir, Semisimple Hopf algebras via geometric invariant theory. Adv. Math. 311 (2017), 61-90.

[9] S. Montgomery, Hopf algebras and their actions on rings. CBMS Regional Conference Series in Mathematics 82. American Mathematical Society, Providence, RI, 1993.

[10] W. Narkiewicz, Elementary and analytic theory of algebraic numbers (Third edition). Springer Monographs in Mathematics. Springer-Verlag, Berlin, 2004.

[11] D. Nikshych, Non group-theoretical semisimple Hopf algebras from group actions on fusion categories. Selecta Math. (N.S.) 14 (2008), 145-161.

[12] D. E. Radford, Hopf algebras. Series on Knots and Everything 49. World Scientific Publishing Co. Pte. Ltd., Hackensack, NJ, 2012.

[13] D. E. Radford, E. J. Taft, and R. L. Wilson, Forms of certain Hopf algebras. Manuscripta Math. 17 (1975), 333-338.

[14] J. Tate and F. Oort, Group schemes of prime order. Ann. Sci. École Norm. Sup. (4) 3 (1970), 1-21.

[15] R. Underwood, The valuative condition and $R$-Hopf algebra orders in $K C_{p^{3}}$. Amer. J. Math. 118 (1996), 701-743.

[16] L. C. Washington, Introduction to cyclotomic fields. Second edition. Graduate Texts in Mathematics 83. Springer-Verlag, New York, 1997.

J. Cuadra: Universidad de Almería, Dpto. Matemáticas. E04120 Almería, Spain

E-mail address: jcdiaz@ual.es 
E. Meir: Department of Mathematics, University of Hamburg, Bundesstrasse 55, 20146 Hamburg, Germany

E-mail address: meirehud@gmail.com 